\title{
Physics of Dynamic Stall Vortex During Pitching Oscillation of Dynamic Airfoil
}

\author{
Binbin Wei ${ }^{1}$ (D) Yongwei Gao ${ }^{1}$ (D) Dong $\mathrm{Li}^{1}$
}

Received: 13 September 2020 / Revised: 6 May 2021 / Accepted: 11 May 2021 / Published online: 29 July 2021

(c) The Author(s) 2021

\begin{abstract}
Dynamic stall, which has a significant effect on the aerodynamic performances of dynamic airfoils, is closely related to the physics of the dynamic stall vortex (DSV). The physics of the DSV on the NACA 0012 airfoil was experimentally studied using unsteady pressure measurements with high time accuracy. The experimental Reynolds number was $\operatorname{Re}=1.5 \times 10^{6}$, and the reduced frequency was $k=0.069$. The propagation of the unsteady pressure field during the dynamic stall process was analyzed in detail. The motion characteristics of the DSV were examined, including its near-wall development characteristics and near-wall evolution velocity. Moreover, the frequency characteristics of the near-wall DSV were studied using wavelet analysis combined with proper orthogonal decomposition (POD) technology. In addition, the effects of the mean angle of attack (AoA) and the amplitude on the DSV motion and frequency characteristics were examined in detail. The effects of the mean AoA on the near-wall DSV strength and the propagation velocity were linear, while the effects of amplitude were nonlinear. The mean AoA and amplitude had a significant influence on the frequency of the leading-edge vortex (LEV) at the initial stage of the DSV development $(x / c=0.10-0.20)$. This work allows the DSV physics to be understood more deeply.
\end{abstract}

Yongwei Gao

gyw630@nwpu.edu.cn

Binbin Wei

weibinbin123@mail.nwpu.edu.cn

Dong Li

ldgh@nwpu.edu.cn

1 School of Aeronautics, Northwestern Polytechnical University, Xi'an, Shaanxi, China 


\section{Graphic abstract}

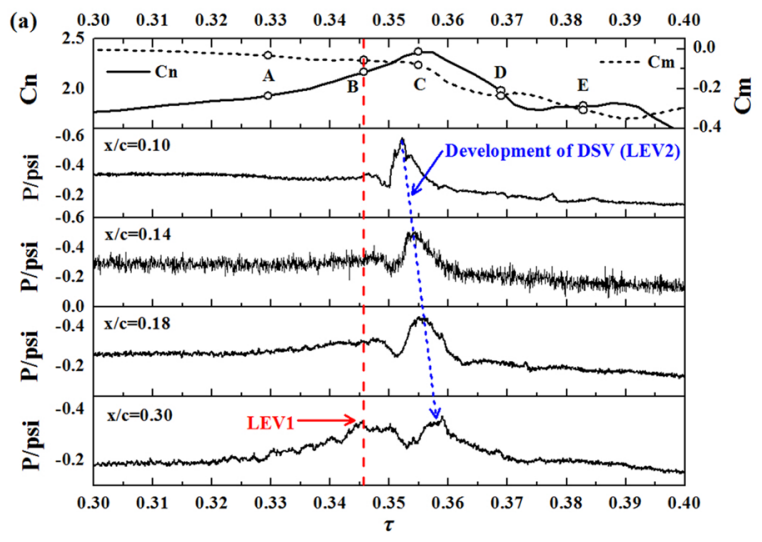

(b) $\quad \begin{array}{lllllllllll}0.30 & 0.31 & 0.32 & 0.33 & 0.34 & 0.35 & 0.36 & 0.37 & 0.38 & 0.39 & 0.40\end{array}$
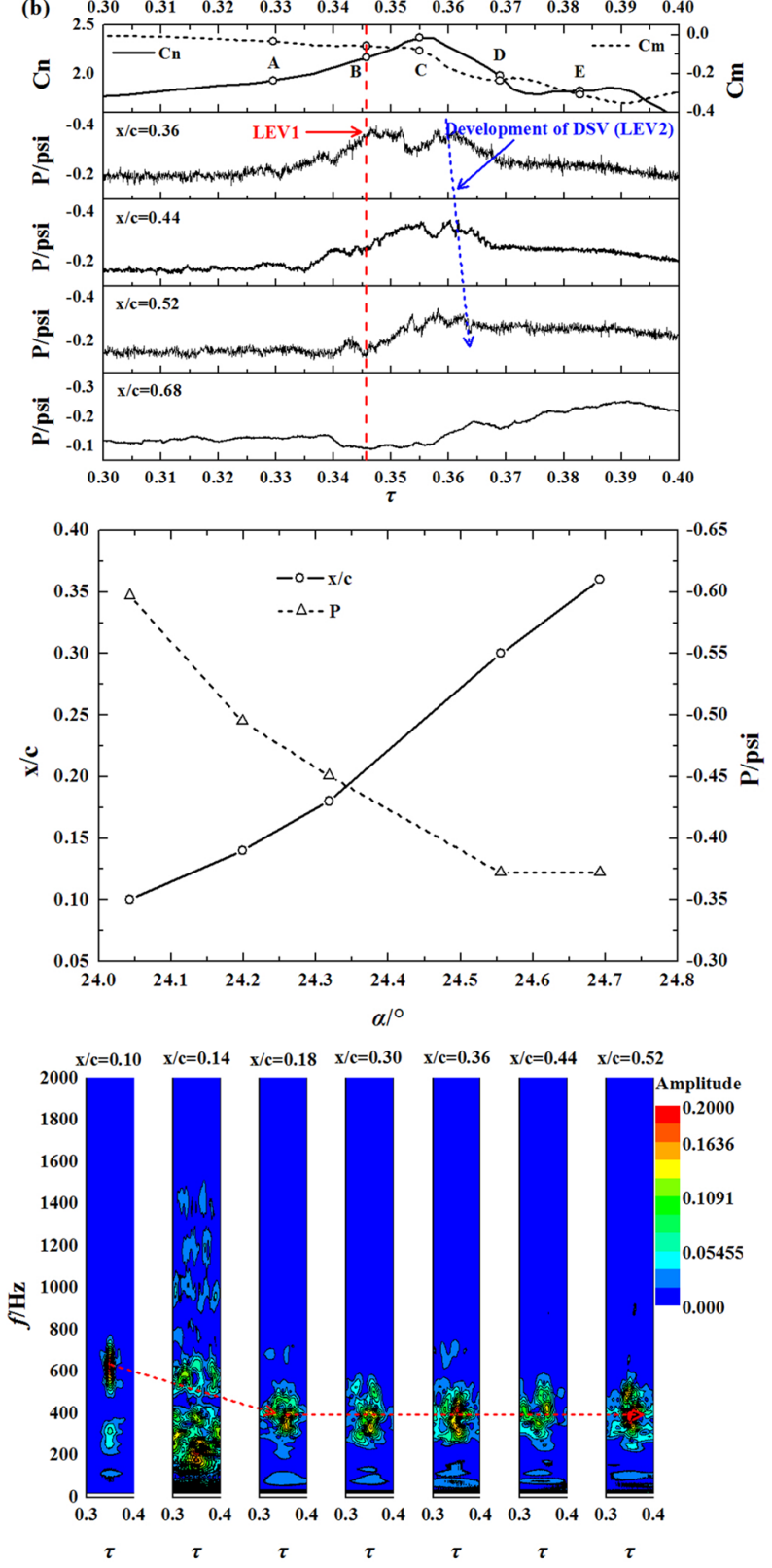

Keywords Dynamic stall vortex · Dynamic stall ·

Time-frequency analysis · Pitching oscillation

\section{Introduction}

The dynamic stall process has a significant effect on the aerodynamic performances of dynamic airfoils [1]. In general, when the airfoil load increases significantly, dynamic stall begins to occur. During dynamic stall, the unsteady characteristics of the flow field increase significantly, and the aerodynamic force and moment undergo nonlinear changes.

It is generally believed that the formation and development of the dynamic stall vortex (DSV) directly affects the dynamic stall process. Many studies of Carr et al. [1-6] greatly enhanced the understanding of the DSV and dynamic stall. Before the start of dynamic stall, a very thin reversed flow area forms at the trailing edge. Under the action of a reverse pressure gradient, the reversed flow area gradually moves to the leading edge. During this process, the flow remains attached. A vortex that results in a strong suction at the leading edge is then formed. A vortex called the leadingedge vortex (LEV) gradually separates from the airfoil surface to form a DSV. As the angle of attack (AoA) increases, the DSV begins to develop toward the trailing edge, resulting in significant torque changes. As the AoA further increases, the DSV separates from the trailing edge of the airfoil eventually.

The evolution of the DSV in the flow field is highly nonlinear and unsteady. As a result, it is difficult to predict or simulate dynamic stall accurately. Therefore, wind tunnel experiments are usually used to study the dynamic stall.

Many scholars have focused on the evolution of the unsteady flow fields during dynamic stall. Mulleners et al. [7] used time-resolved particle image velocimetry (TR-PIV) to study the unsteady flow field during the dynamic stall process. The results showed that the formation of large-scale coherent structures and convection as well as flow separation delay were the most important characteristics of dynamic stall. Wei et al. [8] studied the flow separation characteristics of the S809 airfoil during its pitch oscillation using fluctuating pressures. The results showed that the flow separation advanced and the reattachment was delayed with the increase in the oscillation frequency, resulting in greater hysteresis. Gupta and Ansell [9] used the fluctuating pressure and PIV technology to study the unsteady flow field of the NACA 0012 airfoil in the dynamic stall process. This research focused on the analysis of the development of the laminar separation bubble (LSB) and DSV. The results showed that the center frequency of the leading edge separation bubble was $50 \leq \mathrm{St} \leq 110$. Zou and Lee [10] and Jia et al. [11] studied the development of rotor boundary layer using PIV technology.

The DSV has been experimentally studied by many scholars. Pruski and Bowersox [12] performed a phase-averaged 
PIV experiment on a pitching oscillation airfoil, and compared the transient and phase-averaged characteristics of the DSV. They suggested that in a transient sense, the DSV was formed through multiple vortex structures. Acharya and Metwally [13] measured and analyzed the transient pressure on airfoils during the upstroke process. The study showed that the behavior of vortex structures on the airfoil surfaces were related to the interaction mechanism of the flow field development. The propagation speed of DSV is also a concern of many scholars. McCroskey et al. [14], Lorber and Carta [15], Chandrasekhara and Carr [16], Zakaria et al. [17] and Wang and Zhao [18] all studied the propagation velocity of the DSV, and showed that the propagation velocity of DSV is $0.3 U-0.4 U$.

To understand the interaction mechanism between the DSV and dynamic stall, the flow control of the dynamic stall was also studied. In terms of active control, Karim and Acharya [19] used the leading-edge suction method to control the DSV. The study showed that the formation of the DSV could be effectively inhibited by removing an appropriate amount of the backflow area to prevent its accumulation near the leading edge. In terms of passive control, Geissler et al. [20, 22] used leading edge vortex generators to control dynamic stall. They effectively suppressed the occurrence of the dynamic stall. Ajalli and Mani [21] studied the effect of trailing edge strips (TES) on the wake area of an airfoil during plunging motion. The study showed that when the airfoil oscillated outside the static stall AoA, the wake shape changed significantly under the influence of the LEV and von Kármán vortices. These studies all indicated that the DSV plays a key role in the dynamic stall process.

In addition, the influence of the flow parameters and airfoil shape parameters on dynamic stall were also studied. Wang and Zhao [18] used PIV technology to study the influence of the leading-edge radius of the airfoil on dynamic stall, and the study showed that the strength of the LEV on the airfoil with a large leading edge radius weakened significantly. Lorber and Carta [15] studied the influence of the angular velocity and Mach number on dynamic stall. This study showed that the increase in the angular velocity strengthened the LEV, while the increase in the Mach number weakened it. Geissler et al. [20, 22] studied the effect of nose-drooping, which is also a means of flow control, on supercritical airfoils in dynamic stall control research activities (project ADASYS of DLR), and found that it could effectively improve the dynamic stall characteristics of airfoils. Subsequently, Geissler and Haselmeyer [23] continued to study the influence of flow transitions on the occurrence of dynamic stall and found that the development and diffusion process of a turbulent separation region from the back to the front had a significant impact on the occurrence of dynamic stall.
In spite of these studies, the physics of the DSV are still not fully understood, such as the influence of the mean AoA and the amplitude on the propagation characteristics of the DSV. In this study, the physics of the DSV were experimentally examined using a fluctuating pressure on the NACA 0012 airfoil. The experimental Reynolds number was $\operatorname{Re}=1.5 \times 10^{6}$, and the reduced frequency was $k=0.069$. The near-wall development characteristics and near-wall propagation velocity of the DSV in a typical dynamic stall process $\left(\alpha_{0}=15^{\circ}, A=15^{\circ}\right)$ were studied in detail, and the frequency characteristics of the nearwall DSV were analyzed using wavelet analysis combined with proper orthogonal decomposition (POD) technology. Based on these results, the effects of the mean AoA and amplitude of the AOA on the DSV motion and frequency characteristics were studied in detail. The effect of the mean AoA was linear, while the effect of the amplitude was nonlinear.

\section{Experimental Setup}

The experiment was conducted in the NF-3 wind tunnel at Northwestern Polytechnical University, Xi' an, China. The size of the airfoil test section was $8.0 \mathrm{~m} \times 1.6 \mathrm{~m} \times 3.0 \mathrm{~m}$ (length $\times$ height $\times$ width). The maximum wind speed was $U=130 \mathrm{~m} / \mathrm{s}$ and turbulence intensity was less than $0.05 \%$.

The airfoil dynamic drive mechanism was used to implement the pitching motion of the airfoil model, as shown in Fig. 1. The oscillation frequency could be controlled in the range of $f=0-5 \mathrm{~Hz}$. The maximum angular amplitude was $A_{\max }=15^{\circ}$ and the average AoA was $\alpha=0^{\circ}-360^{\circ}$ with an accuracy of $\varepsilon \leq 6^{\prime}$.

To study the physical characteristics of the near-wall DSV, the transient pressure data on the airfoil surface were

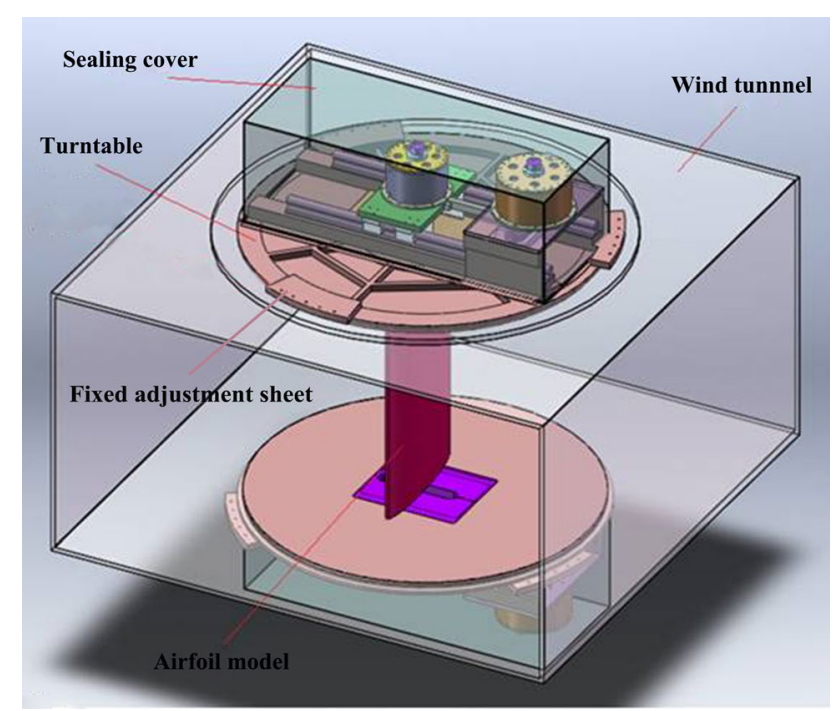

Fig. 1 Schematic diagram of rotary mechanism 


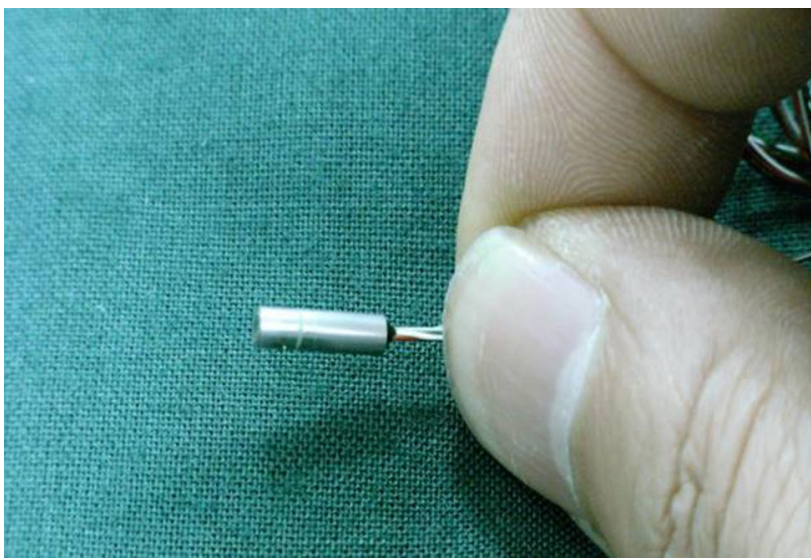

Fig. 2 Pressure sensor

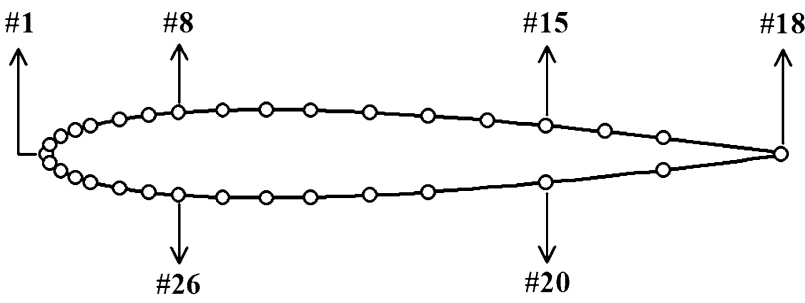

Fig. 3 NACA0012 airfoil and sensor arrangement

collected by a VXI data acquisition system that had 48 acquisition channels. The acquisition precision was 16 bit analog-to-digital (A/D), and the acquisition speed was not less than $100 \mathrm{kHz} / \mathrm{channel}$.

The experimental model was the NACA0012 airfoil with a chord length of $c=700 \mathrm{~mm}$ and the span length of $l=1600 \mathrm{~mm}$. The rotation axis was located at the position of $x / c=0.25 .32$ Kulite XCQ-093 pressure sensors were arranged clockwise along the middle surface of the model $(z / l=0.50)$, including 16 on the upper surface, 14 on the lower surface, and 1 on each of the leading and trailing edges, as shown in Figs. 2 and 3. To capture the LEV structure, 10 sensors were arranged at $x / c \leq 0.30$ on the suction surface. To study the fluctuating characteristics of the LEV, the sampling rate of the experiment was set to $f_{\mathrm{s}}=20 \mathrm{kHz}$ to ensure a sufficiently high time accuracy.

The experimental Reynolds number was $\operatorname{Re}=1.5 \times 10^{6}$, and the free flow velocity was $U=31.7 \mathrm{~m} / \mathrm{s}$. The sinusoidal oscillation frequency of the airfoil was $f=1.0 \mathrm{~Hz}$, and the corresponding reduced frequency was $k=\pi f c / U=0.069$.

The dynamic stall process of the NACA0012 airfoil in a typical state was presented in the study of Carr [1], and the dynamic stall occurred at $\alpha \approx 20^{\circ}-22^{\circ}$. To study the physics of the DSV, dynamic stall should occur within the range of the experimental AoAs. The experimental conditions are shown in Table 1.
Table 1 Experimental condition

\begin{tabular}{|c|c|c|c|c|}
\hline \multirow[t]{2}{*}{ Case } & \multicolumn{2}{|l|}{ Variable } & \multicolumn{2}{|c|}{$\begin{array}{l}\text { Experimental param- } \\
\text { eters }\end{array}$} \\
\hline & $\begin{array}{l}\text { Mean AoA } \\
\alpha_{0}{ }^{\circ}\end{array}$ & $\begin{array}{l}\text { Amplitude of } \\
\text { AoA } A /^{\circ}\end{array}$ & $\mathrm{Re} / 10^{6}$ & $k$ \\
\hline 1 & 5 & 15 & 1.5 & 0.069 \\
\hline 2 & 10 & 15 & 1.5 & 0.069 \\
\hline 3 & 15 & 15 & 1.5 & 0.069 \\
\hline 4 & 15 & 5 & 1.5 & 0.069 \\
\hline 5 & 15 & 10 & 1.5 & 0.069 \\
\hline
\end{tabular}

The experimental conditions are shown in Table 1, and the AoA range included the AoA when dynamic stall occurred. Case1, Case2, and Case 3 were used to study the effect of the mean AoA $\alpha_{0}$ on the physical characteristics of the DSV, and Case1, Case4, and Case5 were used to study the influence of the amplitude $A$.

The feasibility of the phase averaging method and slip window technology (SWT) was discussed in detail in our previous publication [8]. It was found that the phase averaging method was suitable for a low sampling rate and more sampling periods, while the SWT was reasonable when the sampling rate was high and the number of sampling periods was smaller. To ensure sufficient time accuracy, the sampling rate in this experiment was set to $f_{\mathrm{s}}=20 \mathrm{kHz}$. With such a high sampling rate, it was appropriate to use SWT to process the data.

\section{Experimental Results and Data Analysis}

The physical characteristics of the DSV on the NACA0012 airfoil during its pitching oscillation were experimentally studied using surface pressure measurements. The experimental Reynolds number was $\operatorname{Re}=1.50 \times 10^{6}$, and the reduced frequency was $k=0.069$. The dynamic stall process for a typical state $\left(\alpha_{0}=15^{\circ}, A=15^{\circ}\right)$ was analyzed. The development characteristics of the DSV for this typical state were explored. The frequency characteristics of the near-wall DSV were analyzed in detail using wavelet analysis combined with POD technology. Based on these approaches, the effects of the mean AoA and amplitude of the AoA on the physical characteristics of the DSV were studied.

\subsection{Evolution Process of Dynamic Stall}

Taking the case of $\alpha_{0}=15^{\circ}, A=15^{\circ}$ as an example, the pressure evolution process of dynamic stall in this typical state is analyzed in this section. 

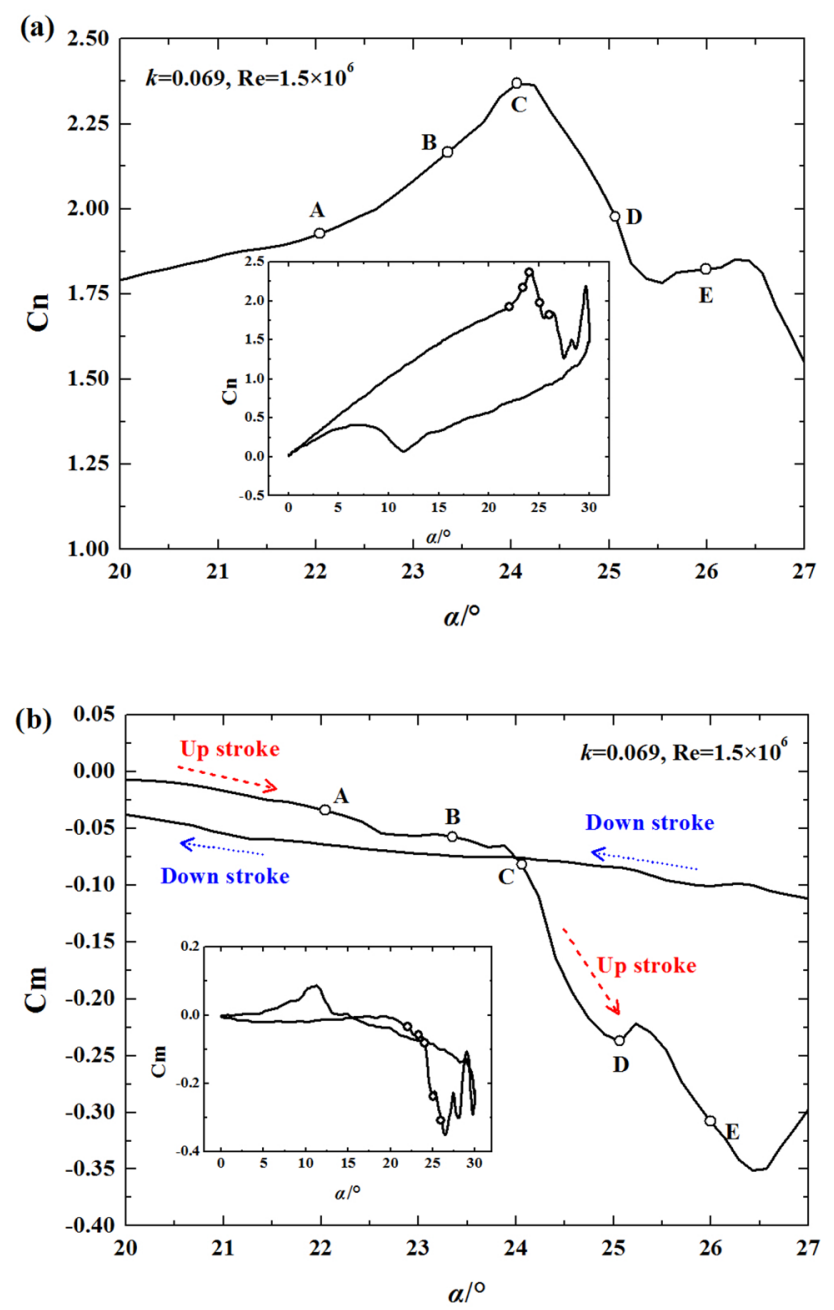

Fig. 4 Normal force and moment coefficients

The normal force and moment coefficients for the typical state $\left(\alpha_{0}=15^{\circ}, A=15^{\circ}\right)$ are shown in Fig. 4. In the high-AoA phase $\left(\alpha \approx 22^{\circ}-26^{\circ}\right)$ during upstroke, the slope of the normal force line began to increase significantly at AoA $A$, and the normal force and the moment coefficients all decreased sharply at AoA $C$, indicating that a noticeable dynamic stall occurred.

The pressure coefficients during upstroke at typical positions $(x / c=0.10$ and $x / c=0.52)$ are shown in Fig. 5. Compared with the overall force and moment, the curve of the pressure coefficients can represent the dynamic stall phenomenon more clearly.

The unsteady pressure evolution process of the dynamic stall is shown in Fig. 6. The attached vortex structure at the leading edge is called LEV. The vortex structure with wider influence range on the suction surface is called DSV, though it is essentially a LEV. The development process of the LEV and the DSV is clear. Under the effect of an adverse pressure gradient (AoA $B$ ), the first LEV, called LEV1, was

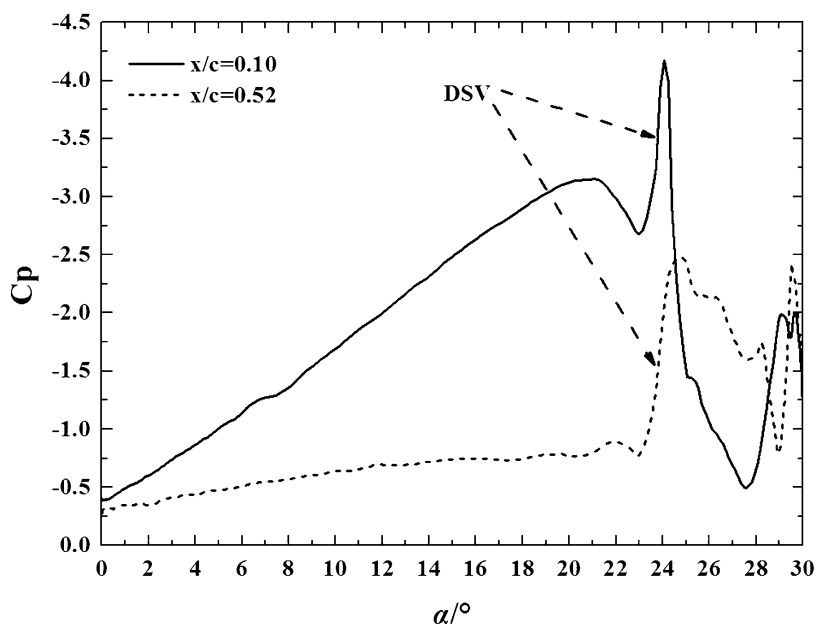

Fig. 5 Pressure coefficients at typical locations

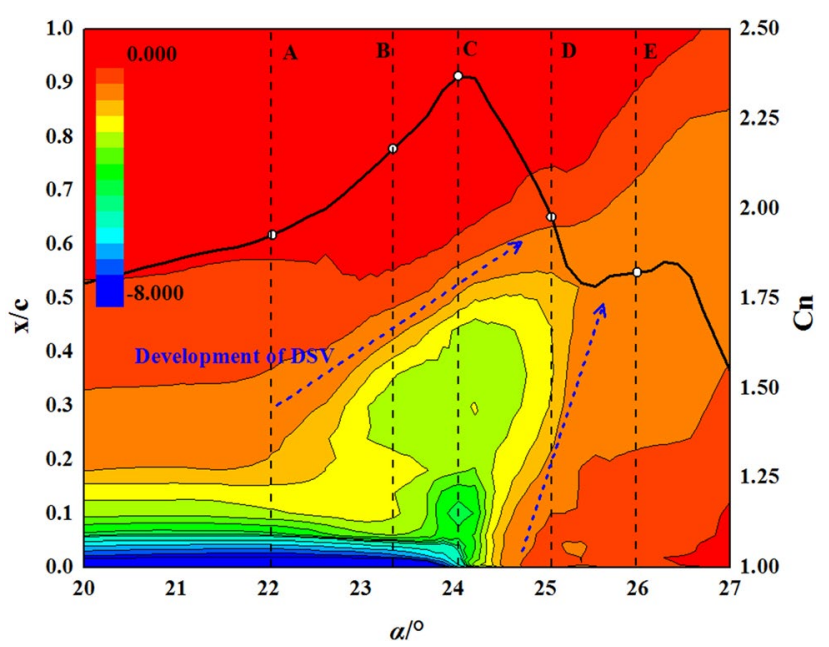

Fig. 6 Pressure field evolution in dynamic stall

formed at $x / c=0.20-0.30$. As the AoA increased to $C$, a second LEV, called LEV2, was then formed in front of LEV1 $(x / c=0.10)$. These LEVs caused a strong suction peak. As a result, the normal force coefficient increased significantly. As the AoA further increased (AoA $D$ and $E$ ), the LEV2 gradually developed toward the trailing edge to form a DSV. During this development process, the normal force and the moment coefficients all decreased drastically.

The pressure distributions at several typical AoAs are shown in Fig. 7. In the transition from $A$ to $C$ (the process of increasing the slope of the normal force coefficient significantly), LEV1 with a strength of $C_{\mathrm{p}}=-3.16$ was generated at the positions of $x / c=0.24-0.36$ at AoA $B$. As the AoA gradually increased to $C$, LEV2 formed at the position $x / c=0.10$ with a strength of $C_{\mathrm{p}}=-4.17$, which was consistent with the study of Worasinchai et al. [24]. In the phase of $C-E$, LEV2 gradually developed backward to form 


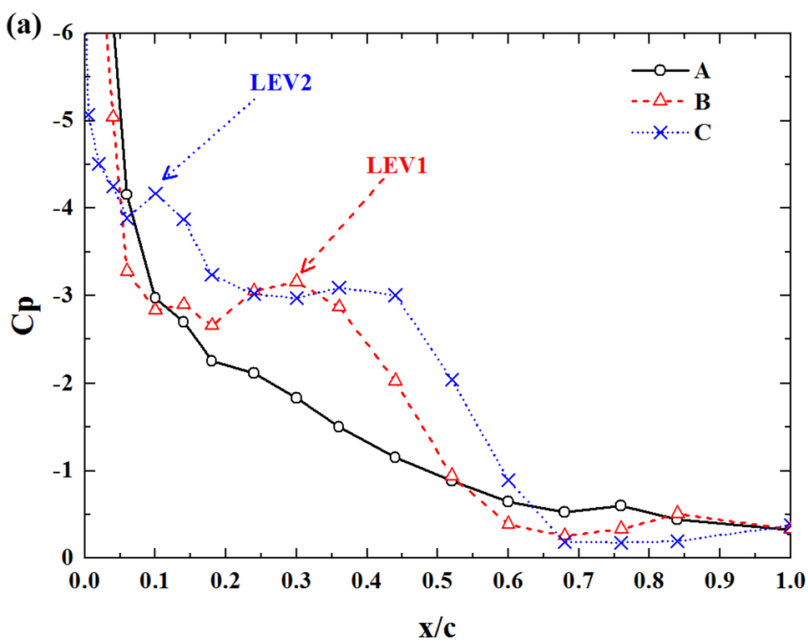

(b)

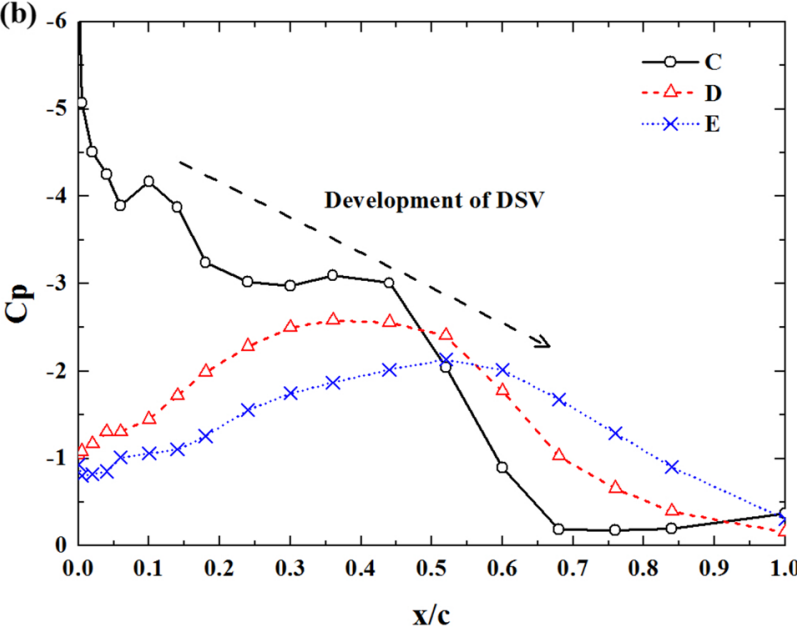

Fig. 7 Pressure evolution at typical AoAs. a Evolution of LEVs. b Development of DSV (LEV2)

a DSV as the AoA further increased. In this process, the DSV moved backward along the airfoil surface, resulting in a sharp decrease in the moment coefficient, which is shown in Fig. 4b.

Many scholars have discovered these LEVs in their studies. Bourdet et al. [25], Choudhuri and Knight [26], and Geissler and Haselmeyer [23] all found such LEVs in the computational fluid dynamics (CFD) results. Gupta and Ansell [9] and Geissler et al. [20, 22] also found such LEVs in the pressure distributions of the experimental data. Sharma and Poddar [27] found LEVs through the so-called $\mathrm{Cn}-\mathrm{Cm}$ cross-correlation plots, which were obtained experimentally. In addition, Li et al. [28] found LEVs in CFD and PIV results. As discussed in this section, the formation, convective development, and shedding process of these LEVs involve a complex aerodynamic performance to the airfoil.

\subsection{Motion Features of DSV}

The motion characteristics of the DSV have a significant influence on the dynamic stall. The case of $\alpha_{0}=15^{\circ}, A=15^{\circ}$ is again used as an example to investigate the near-wall motion characteristics of the DSV.

It was worth noting that the original pressure collected by the differential pressure sensors in this experiment was $P_{\mathrm{c}}=P-P_{0}$, and the pressure coefficient is defined as follows:

$C_{\mathrm{p}}=\frac{P-P_{\infty}}{q}=\frac{P-P_{0}+q}{q}=\frac{P_{\mathrm{c}}}{q}+1$,

where $P$ is the static pressure on the airfoil surface, $P_{0}$ is the total pressure, $P_{\infty}$ is the static pressure of incoming flow, and $q$ is the dynamic pressure. There is a linear relationship between the original pressure $P_{\mathrm{c}}$ collected by the differential pressure sensors and the pressure coefficient $C_{\mathrm{p}}$. Therefore, the original pressure data $P_{\mathrm{c}}$ collected by the sensors were used when analyzing the motion characteristics and frequency characteristics of the DSV below.

The pressure evolution at different locations in the process of dynamic stall is presented in Fig. 8. The horizontal ordinate is the dimensionless time $\tau$ which is normalized using the oscillation period $T$ by $\tau=t / T$. The development of the LEV and DSV could be clearly observed in the figure. The DSV moved along the wall, resulting in significantly reduced pressure near the wall.

The minimum pressure value measured at each position (Fig. 8) was taken as the strength of the DSV, and the corresponding AoA was the AoA when the DSV developed. The development characteristics of the DSV were investigated, as shown in Fig. 9.

Figure 9 shows that the DSV moved backward gradually, and its strength gradually decreased with the increase in the AoA, indicating that while the DSV developed backward, it also gradually separated from the airfoil surface.

The near-wall development velocity of the DSV was also investigated, as shown in Fig. 10. The horizontal coordinate is the dimensionless time $\tau$ mentioned earlier, and the ordinate is the dimensionless position of the DSV. The circle in the figure is the experimental result, and the dashed line is the linear fit result based on the following equation:

$x / c=K \tau+b$.

The DSV propagation velocity along the wall can be calculated using the following equation:

$V=\frac{\Delta S}{\Delta t}=\frac{\Delta(x / c) \cdot c}{\Delta \tau \cdot T}=\frac{\Delta(x / c)}{\Delta \tau} \cdot c \cdot f=K \cdot c \cdot f$, 

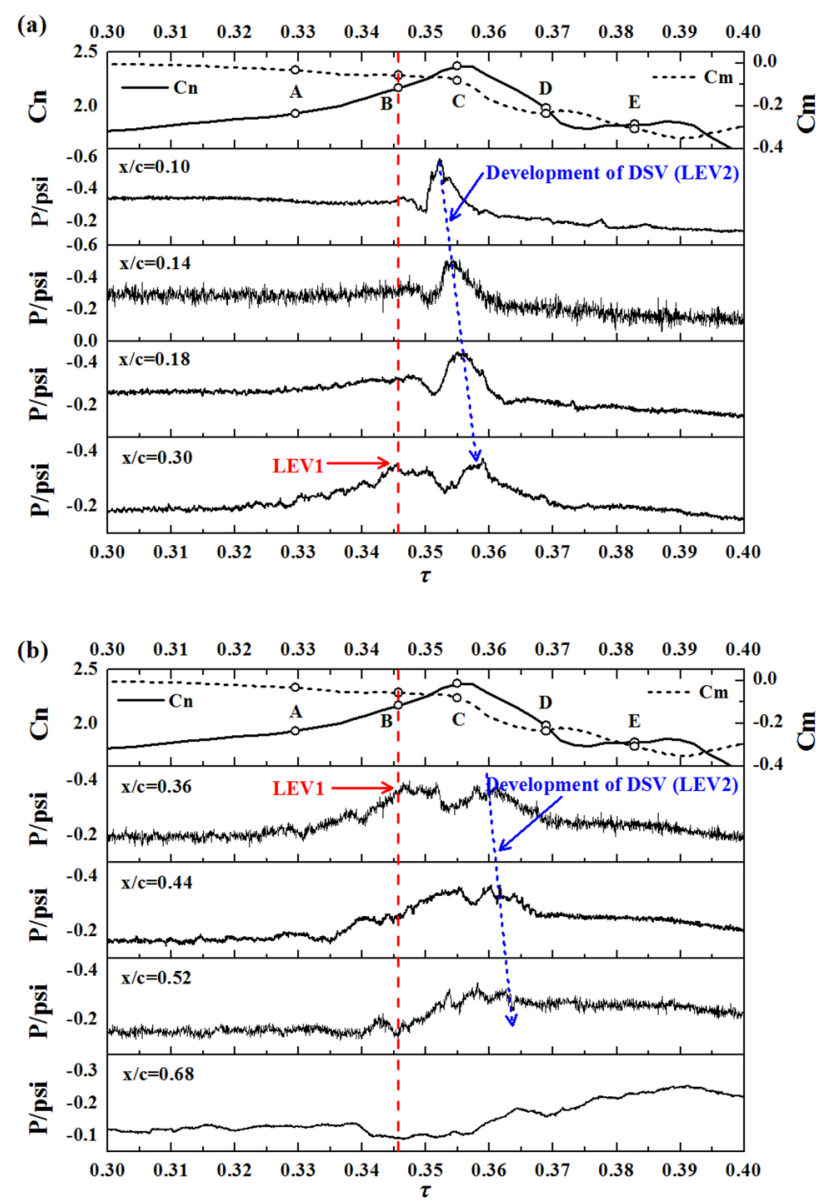

Fig. 8 Original pressure data at different locations. a $x / c=0.10-0.30$. b $x / c=0.36-0.68$

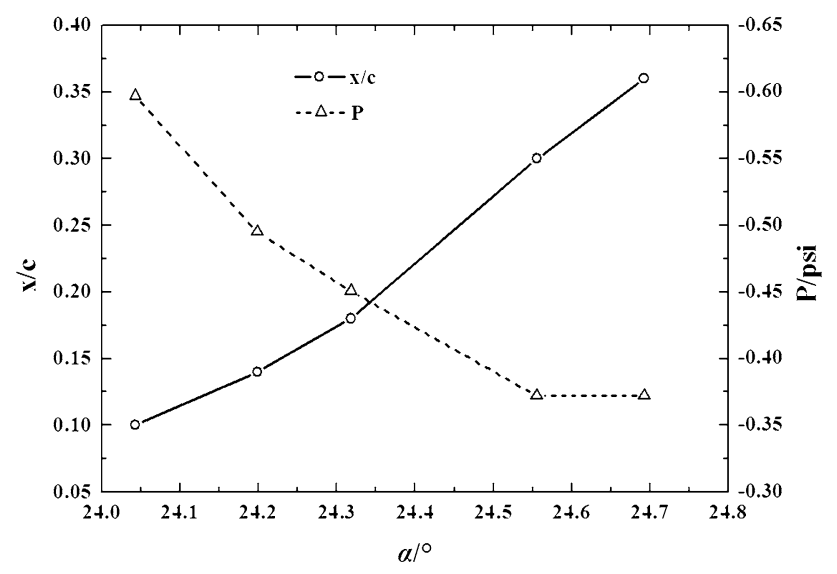

Fig. 9 DSV development characteristics

where the $K$ is the slope of the straight line in Eq. (2), $c$ is the chord length of the airfoil, $T$ is the oscillation period, and $f$ is the airfoil oscillation frequency, which is defined as $f=1 / T$.

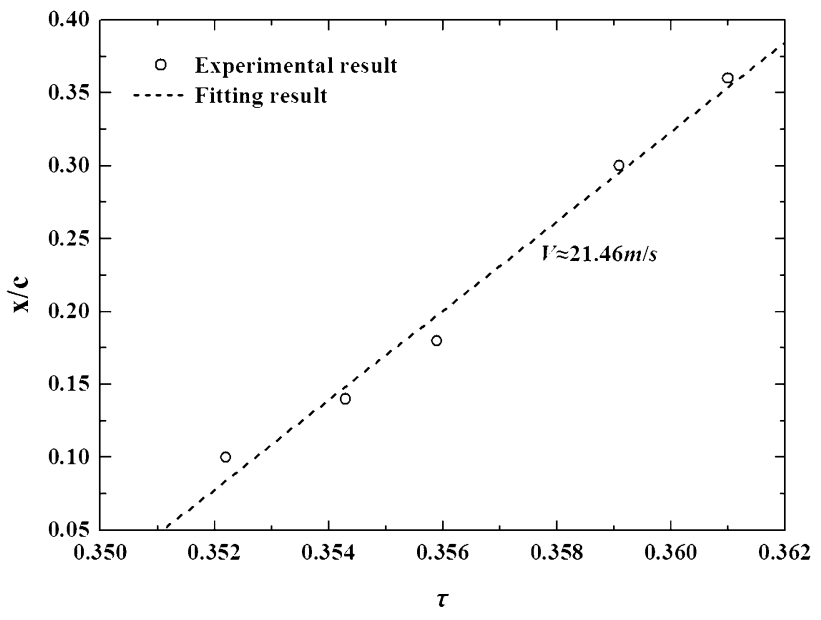

Fig. 10 DSV development velocity

As shown in Fig. 10, the near-wall propagation velocity of the DSV in this case was $V \approx 21.46 \mathrm{~m} / \mathrm{s}$, based on the chord length of the experimental model of $c=700 \mathrm{~mm}$ and the sinusoidal oscillation frequency of $f=1.0 \mathrm{~Hz}$.

\subsection{Frequency Features of DSV}

To obtain the frequency characteristics of the near-wall DSV, the time-frequency analysis of the fluctuating pressure on the airfoil surface was performed. To balance the time and frequency scales, the continuous wavelet transform (CWT) was used to analyze the pressure signal. A wavelet basis function is a set of orthogonal bases in which frequency components of a certain bandwidth are distributed near the center frequency. The complex Morlet wavelets are used as the wavelet basis functions for the CWT. The complex Morlet wavelet expression is as follows:

$\psi(t)=\frac{1}{\sqrt{\pi f_{\mathrm{b}}}} e^{i 2 \pi f_{\mathrm{c}} t} e^{-t^{2} / f_{\mathrm{b}}}$,

where $f_{\mathrm{b}}$ is a bandwidth parameter and $f_{\mathrm{c}}$ is a wavelet center frequency.

The state of $\alpha_{0}=15^{\circ}, A=15^{\circ}$ was still taken as an example to analyze the time-frequency characteristics of DSV.

The time-frequency diagram of wavelet analysis of the original fluctuating pressure at $x / c=0.10$ is presented in Fig. 11. When the LEV was generated, the original pressure data showed characteristics that were significantly different than those of the pressure data obtained at other times. The interval between LEV1 and LEV2 was $\Delta \tau=0.0056$ indicating, that the frequency of the LEVs was $f=179 \mathrm{~Hz}$, which corresponded to the low-frequency characteristics in Fig. 11. The frequency characteristics of the LEVs were 


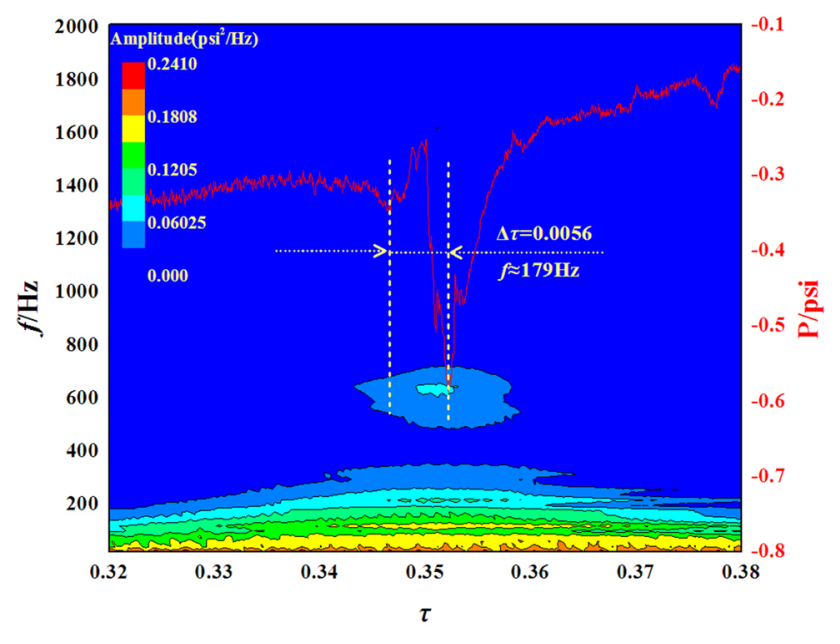

Fig. 11 Time-frequency diagram of original pressure at $x / c=0.10$

extremely evident, and the fluctuating characteristics of the LEV itself would be submerged in many cases.

To investigate the frequency characteristics of the LEV, POD $[29,30]$ technology was used to analyze the original pressure data.

The original pressure time series at a certain position is denoted as follows:

$P=\left[p_{1}, p_{2}, \ldots p_{N}\right]^{T}$,

where $N$ represents the data quantity. A pressure field $\{P\}$ is constructed as follows:

$\{P\}=\left[\begin{array}{cccc}p_{1} & p_{2} & \cdots & p_{M} \\ p_{2} & p_{3} & \cdots & p_{M+1} \\ \cdots & \cdots & \cdots & \ldots \\ p_{N-M+1} & p_{N-M+2} & \cdots & p_{N}\end{array}\right]$,

where $M$ is the data dimension. In this way, the pressure time series $P$ at a certain position can be transformed into an $m$-dimensional pressure field $\{P\}$.

For such a pressure field $\{P\}$, one-dimensional data can be expressed by the following equation:

$P_{i}=\sum_{j=1}^{j=M} a_{i, j} \varphi_{j}$,

where $\varphi$ is a group of orthogonal bases. This method was implemented using the snapshots method proposed by Sirovich [31], and the data dimension $M$ was taken as $M=100$.

The 100-order modes were arranged from high to low in terms of the energy. The POD modal energy and cumulative energy distributions of the pressure sequence in

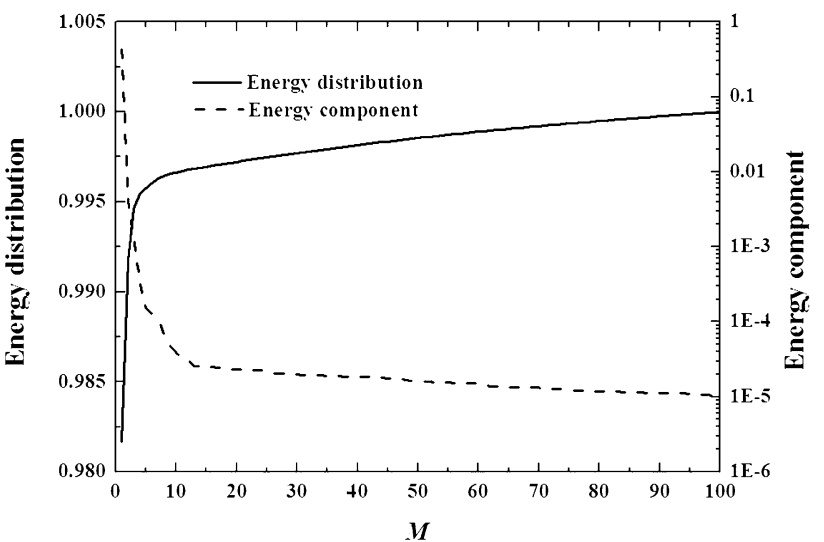

Fig. 12 POD modal energy distribution at position of $x / c=0.10$

Fig. 11 are presented in Fig. 12. The energy of the first mode occupied $98.2 \%$ of the total energy, and the energy of the first six modes occupied $99.6 \%$ of the total energy.

The comparison between the original fluctuating pressure and the POD modes is shown in Fig. 13. The first mode reflected the overall trend of the fluctuating pressure, while the higher mode reflected the fluctuating characteristics of the flow.

To better observe the pressure morphology of the LEV, the pressure near the LEV in Fig. 13 is presented in Fig. 14. The low-order modes (first four orders) simulated the overall morphology of the LEV, while the higher order modes (5th-8th orders) showed the fluctuating characteristics of the LEV. Therefore, the time-frequency analysis of the POD mode could better analyze the flow information.

The time-frequency analysis diagram of several different POD modes (3rd-6th orders) is shown in Fig. 15. The 3rd and 4th modes were still dominated by the frequency of $f \approx 179 \mathrm{~Hz}$ which was the frequency at which the LEVs swept this position, and it was not the feature frequency of the LEV itself. The 5th and 6th modes contained relatively high-frequency characteristics. This frequency characteristic was actually the characteristic frequency of the LEV, which can be seen from the comparison between Figs. 11 and 14 .

The time-frequency diagram of the pressure, which was analyzed using the 5th mode at different positions on the upper surface of the airfoil, is presented in Fig. 16. The characteristic frequency decayed at the early stage (from $f \approx 600$ to $400 \mathrm{~Hz}$ ) during the development of the DSV. When the DSV developed to a certain position, the characteristic frequency of the DSV almost remained unchanged, which was $f \approx 400 \mathrm{~Hz}$. With reference to Fig. 9, the attachment effect of the vortex made it very strong in the early stage of the development of the DSV. With the development of the DSV, it gradually separated from the airfoil surface. The characteristic frequency of the vortex core was high $(f \approx 600 \mathrm{~Hz})$, 

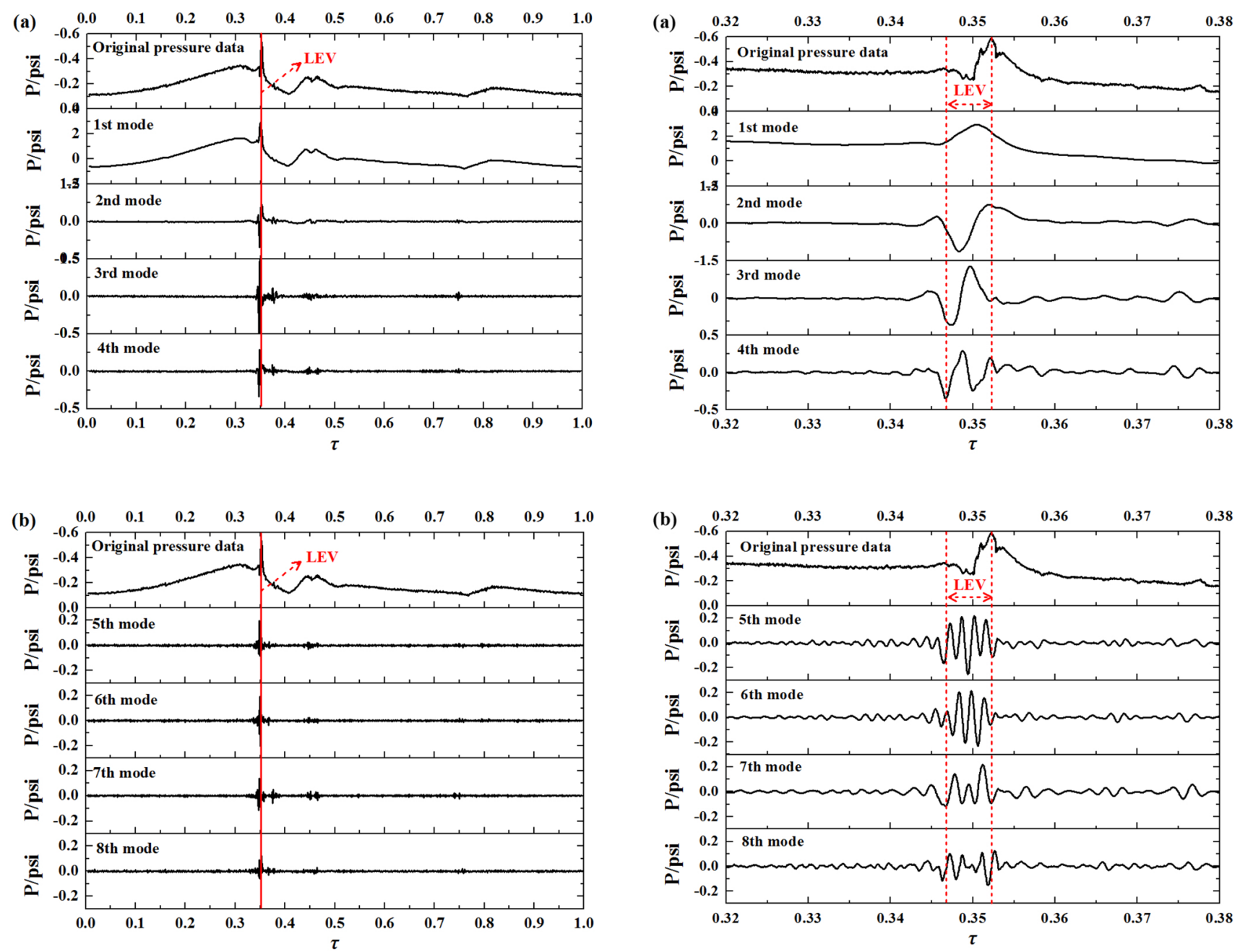

Fig. 13 Comparison of original fluctuating pressure and POD modes at the position of $x / c=0.10$. a 1 st -4 th mode. $\mathbf{b}$ 5th-8th mode

Fig. 14 Comparison of original fluctuating pressure and POD modes at the position of $x / c=0.10$. a 1 st -4 th mode. $\mathbf{b}$ 5th-8th mode

while the characteristic frequency around the vortex core was low $(f \approx 400 \mathrm{~Hz})$.

This section provides a filtering method. For Eq. (7), the reconstruction results of the first $M_{1}$-order modes can be regarded as the trend line or mean value of the original data, while the reconstruction results of the higher order modes after the $M_{1}$-order mode can be regarded as the higher order fluctuation, which can be expressed as follows.

$P_{i}=\sum_{j=1}^{j=M_{1}} a_{i, j} \varphi_{j}+\sum_{j=M_{1}+1}^{j=M} a_{i, j} \varphi_{j}=\bar{P}_{i}+\widetilde{P}_{i}$.

In this study, $M_{1}$ was set to $M_{1}=3$, and the pressure data at $x / c=0.10$ was processed.

The comparison between the original data and the reconstructed results at $x / c=0.10$ is shown in Fig. 17, where the black, red, and blue colors represent the original data, the

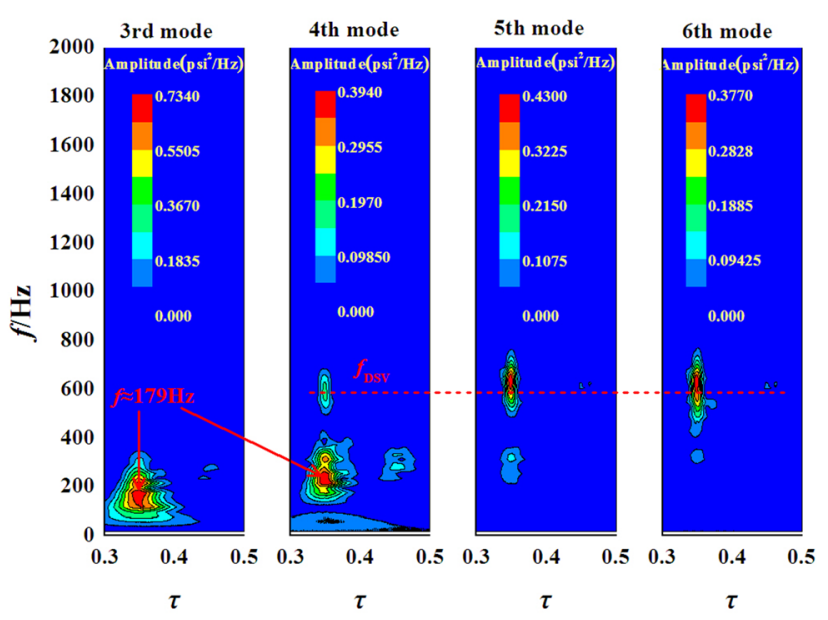

Fig. 15 Time-frequency analysis of different POD modes at $x / c=0.10$ 


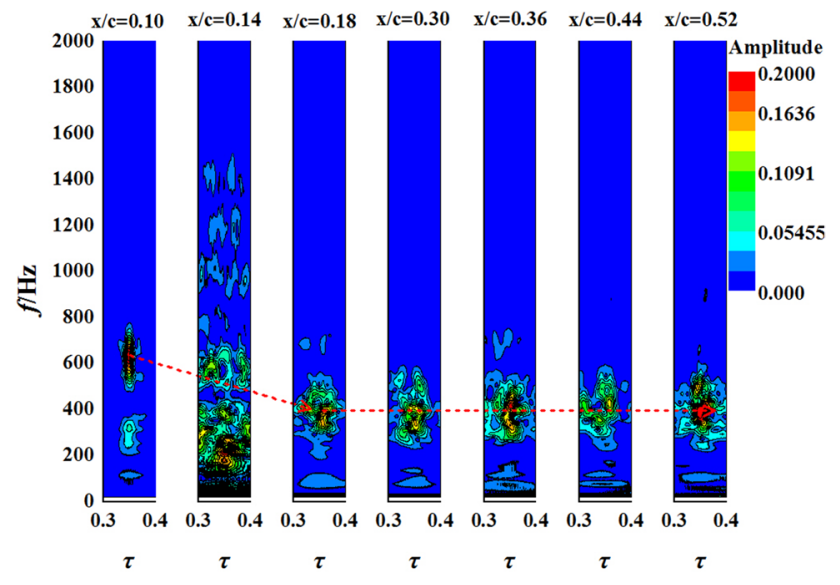

Fig. 16 Time-frequency analysis of pressure information at different positions

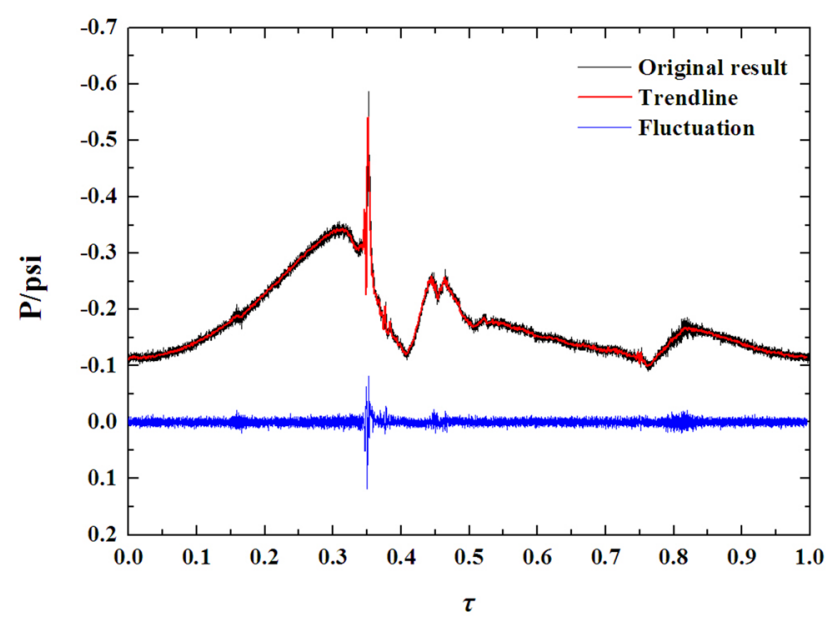

Fig. 17 Original data and reconstruction results at $x / c=0.10$

low-order trend line, and the high-order fluctuation, respectively. The reconstruction results of the first three modes could fully capture the trend of the original data, while the higher order mode reconstruction results could well reflect the higher order fluctuations of the original data.

\subsection{Effect of Mean AoA on DSV}

The case of $\operatorname{Re}=1.5 \times 10^{6}, k=0.069, A=15^{\circ}$ was used to analyze the effect of the mean AoA $\alpha_{0}$ on the aerodynamic characteristics of the airfoil and the DSV motion characteristics.

The effects of the mean AoA on the aerodynamic force and moment coefficients are shown in Fig. 18. The mean AoA had a significant impact on the dynamic stall. As the mean AoA increased, the dynamic stall characteristics became more evident: the maximum normal force coefficient increased, and the minimum moment coefficient decreased.
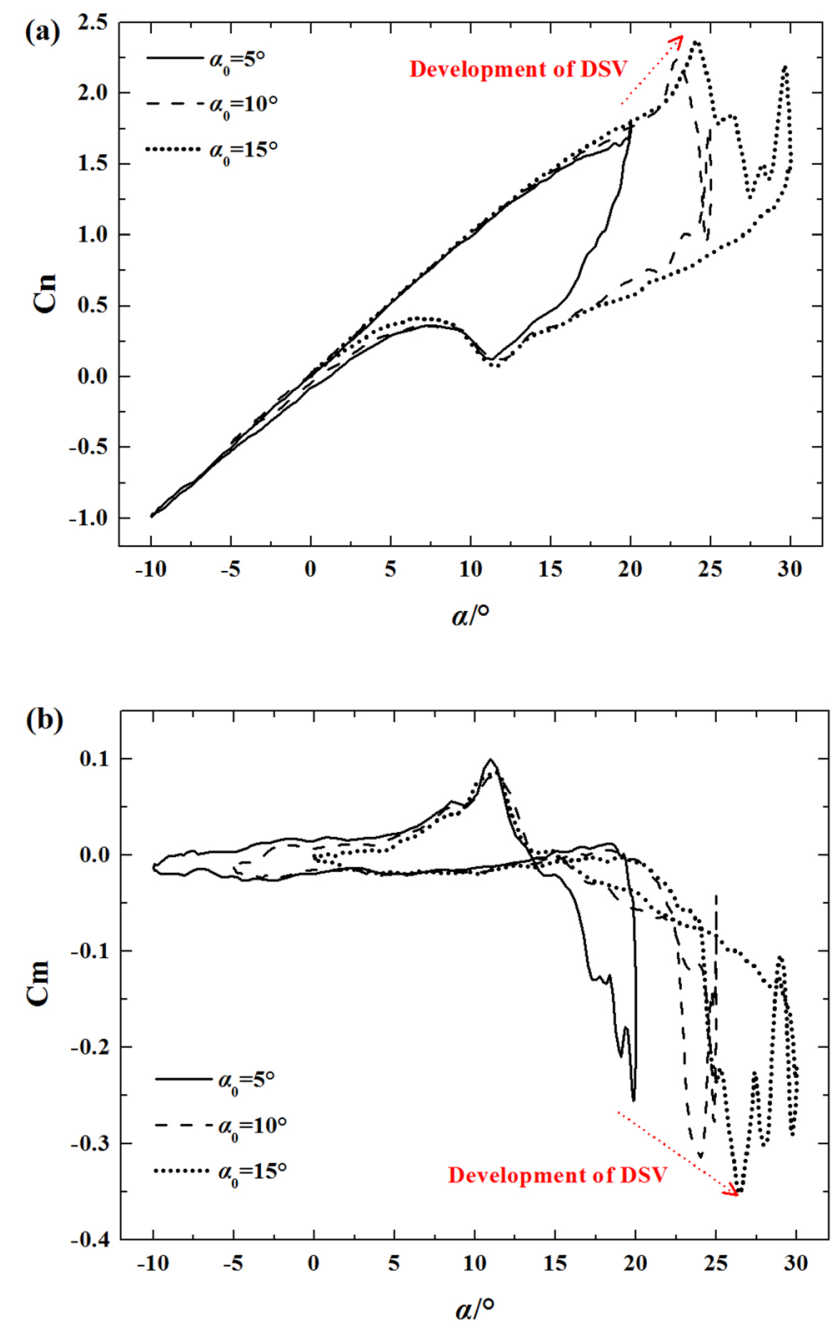

Fig. 18 Effects of mean AoA on aerodynamic force and moment coefficients. a Cn. b Cm

The DSV propagation results for $\alpha_{0}=5^{\circ}$ and $\alpha_{0}=10^{\circ}$ are presented in Figs. 19 and 20, respectively. As evident from the comparison in Fig. $8\left(\alpha_{0}=15^{\circ}\right)$, the generation time and development of the DSV were significantly affected by the mean AoA. The occurrence of dynamic stall is closely related to the AoA. When the mean AoA was small as shown in Fig. $19\left(\alpha_{0}=5^{\circ}\right)$, the phase of dynamic stall was later; when the mean AoA was large, as shown in Fig. $8\left(\alpha_{0}=15^{\circ}\right)$, the dynamic stall occurred earlier in the phase; when the mean AoA was between the two, as shown in Fig. $20\left(\alpha_{0}=10^{\circ}\right)$, the dynamic stall also occurred in the middle of the two phases. In addition to the occurrence time of DSV, the DSV intensity and propagation speed under different mean AoAs are also significantly different, which will be discussed in detail below. In addition to the differences, the three cases with different mean AoAs also have something in common, that is, the DSV development pattern is similar. At the position of the airfoil suction surface $x / c \leq 0.30$, the suction peak brought by 


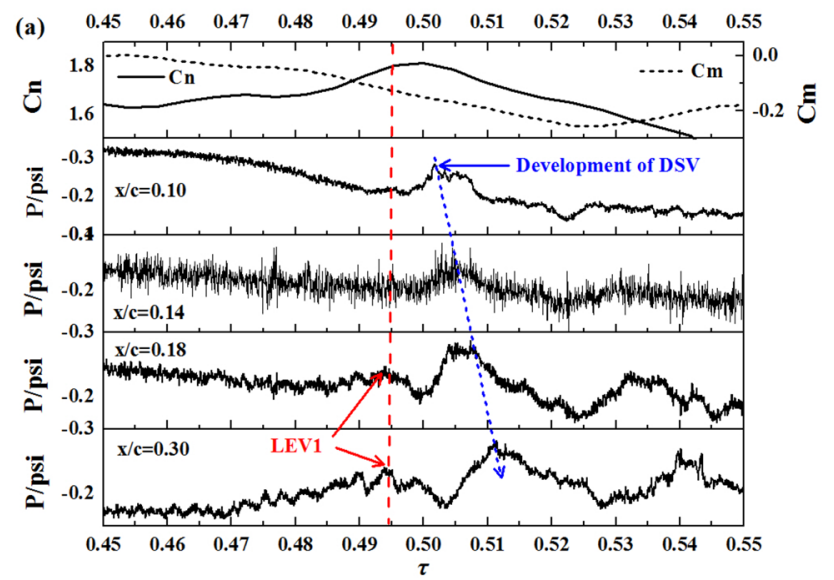

$\begin{array}{llllllllllll}\text { (b) } & 0.45 & 0.46 & 0.47 & 0.48 & 0.49 & 0.50 & 0.51 & 0.52 & 0.53 & 0.54 & 0.55\end{array}$

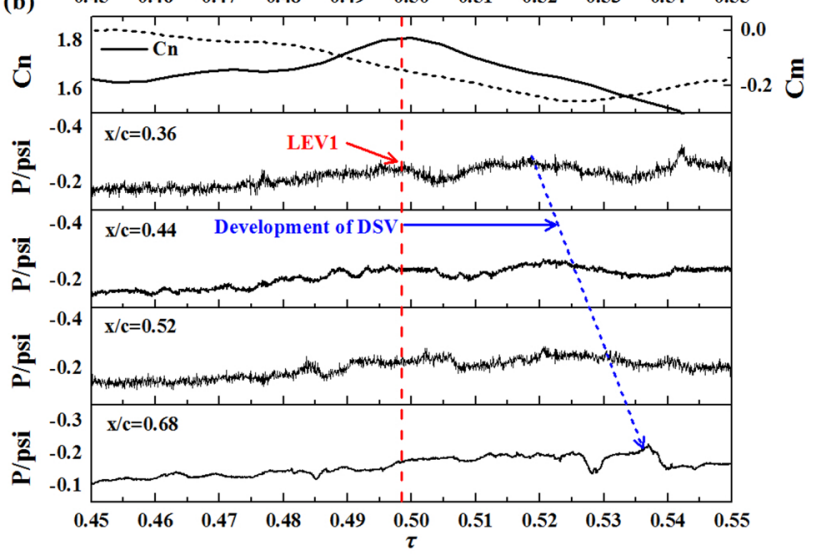

Fig. 19 DSV propagation for $\alpha_{0}=5^{\circ}$. a $x / c=0.10-0.30$. b $x / c=0.36-$ 0.68

DSV is very significant, and the duration of the suction peak is shorter; at the position $x / c>0.30$, the suction peak brought by DSV lasts for a long time.

The effects of the mean AoA on the DSV strength are shown in Fig. 21. With the increase in the average AoA, the strength of the near-wall DSV increased gradually, causing the maximum normal force and minimum moment coefficients to increase and decrease, respectively, which is evident in Fig. 18.

The effects of the mean AoA on the velocity of the DSV evolution on the suction surface were investigated, as shown in Fig. 22. The effect of the mean AoA on the DSV propagation velocity along the near wall surface was almost linear. When the mean AoA was small $\left(\alpha_{0}=5^{\circ}\right)$, the DSV propagated at a lower velocity along the suction surface, which was $V=11.60 \mathrm{~m} / \mathrm{s}$. With the increase in the mean AoA, the propagation speed increased. When the average AoA reached $\alpha_{0}=15^{\circ}$, the DSV propagation velocity along the airfoil wall surface could increase to $V=21.46 \mathrm{~m} / \mathrm{s}$. The free flow with a speed of $U=31.7 \mathrm{~m} / \mathrm{s}$ was used to perform the normalization, and the normalized speeds when the mean AoAs were $\alpha_{0}=5^{\circ}$, $10^{\circ}$, and $15^{\circ}$ were $0.37 U, 0.47 U$, and $0.68 U$, respectively.
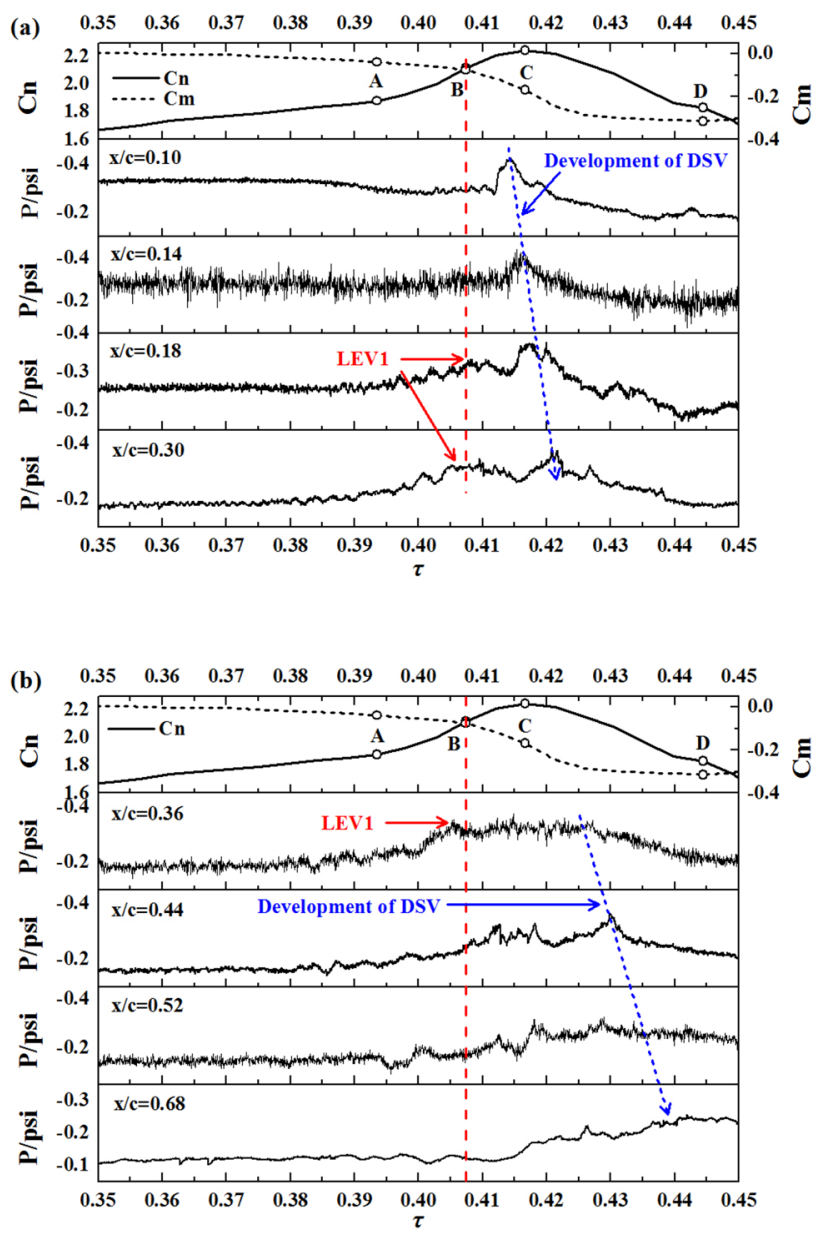

Fig. 20 DSV propagation for $\alpha_{0}=10^{\circ}$. a $x / c=0.10-0.30$. b $x / c=0.36-0.68$

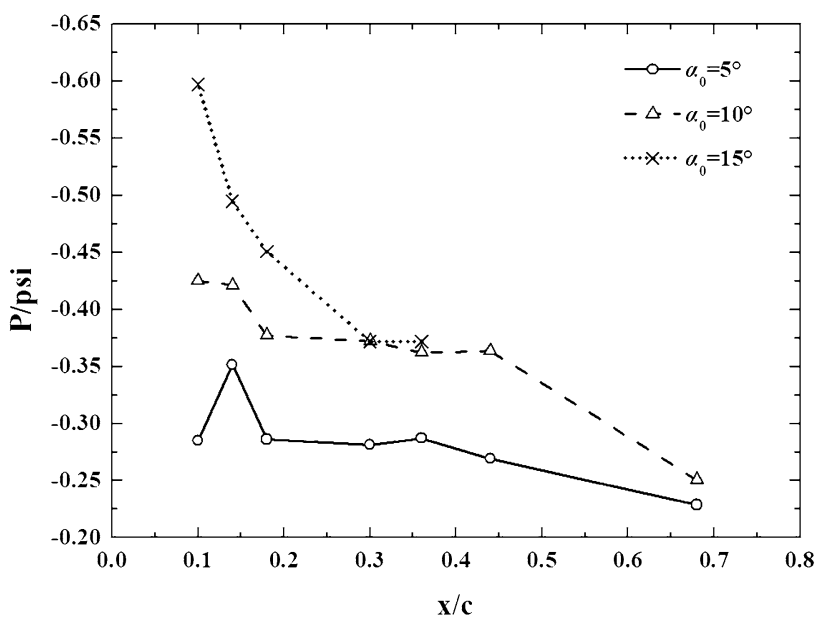

Fig. 21 Effects of mean AoA on DSV strength

The characteristic frequency of the DSV was analyzed using the method presented in Sect. 3.3. The pressure data at each measurement point were analyzed using the POD method with 


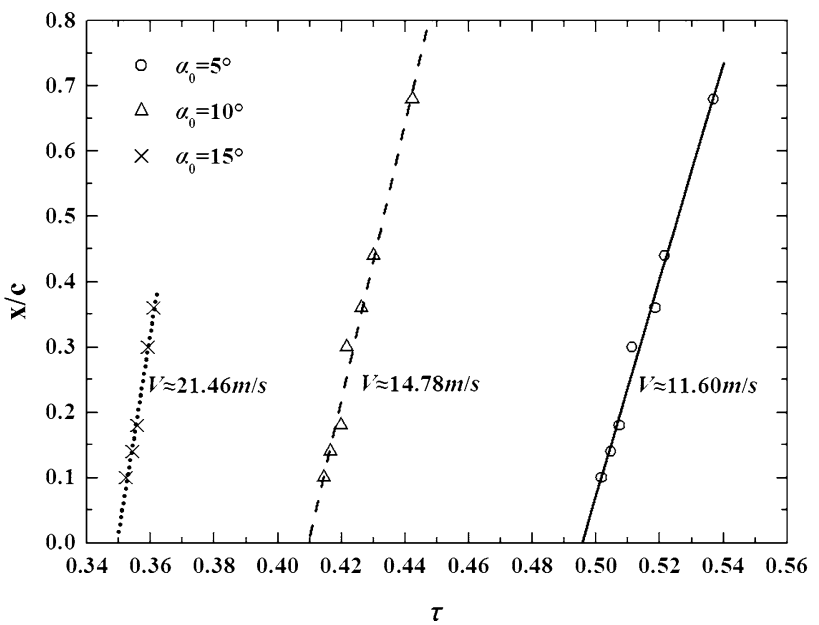

Fig. 22 Effects of mean AoA on DSV evolution velocity

an order of $M=100$, and the 5th mode of each pressure data point was used for the time-frequency analysis. The effects of the average AoA at typical positions on the DSV characteristic frequency at $x / c=0.10$ and $x / c=0.18$ are shown in Fig. 23a, b, respectively. The average AoA had a significant effect on the characteristic frequency of the fluctuating pressure at $x / c=0.10$. This position was at the early stage of the DSV development, called the LEV formation stage which was determined in the previous analysis. At this stage, with the increase in the average AoA, the characteristic frequency of the LEV gradually increased. When the LEV moved backward and developed into the DSV, the influence of the mean AoA on the DSV characteristic frequency gradually weakened.

The effects of the average AoA on the near-wall DSV characteristic frequency are shown in Fig. 24. The average AoA had a significant effect on the characteristic frequency at the early development stage of the DSV (LEV formation stage, $x / c=0.10-0.20$ ). With an increase in the average AoA, the characteristic frequency of the LEV increased gradually. At the later stage of DSV development $(x / c>0.20)$, the average AoA had little effect on the characteristic frequency of the DSV, which remained at $f \approx 400 \mathrm{~Hz}$.

\subsection{Effect of AoA Amplitude on DSV}

The case of $\operatorname{Re}=1.5 \times 10^{6}, k=0.069, \alpha_{0}=15^{\circ}$ was used to analyze the influence of amplitude of AoA $A$ on the aerodynamic characteristics of the airfoil and DSV motion characteristics.

The effects of the amplitude of the AoA on the aerodynamic force and moment coefficients are shown in Fig. 25. The amplitude had a significant effect on the dynamic stall. As the amplitude increased, the maximum normal force coefficient gradually increased, and the minimum moment coefficient first decreased and then increased.
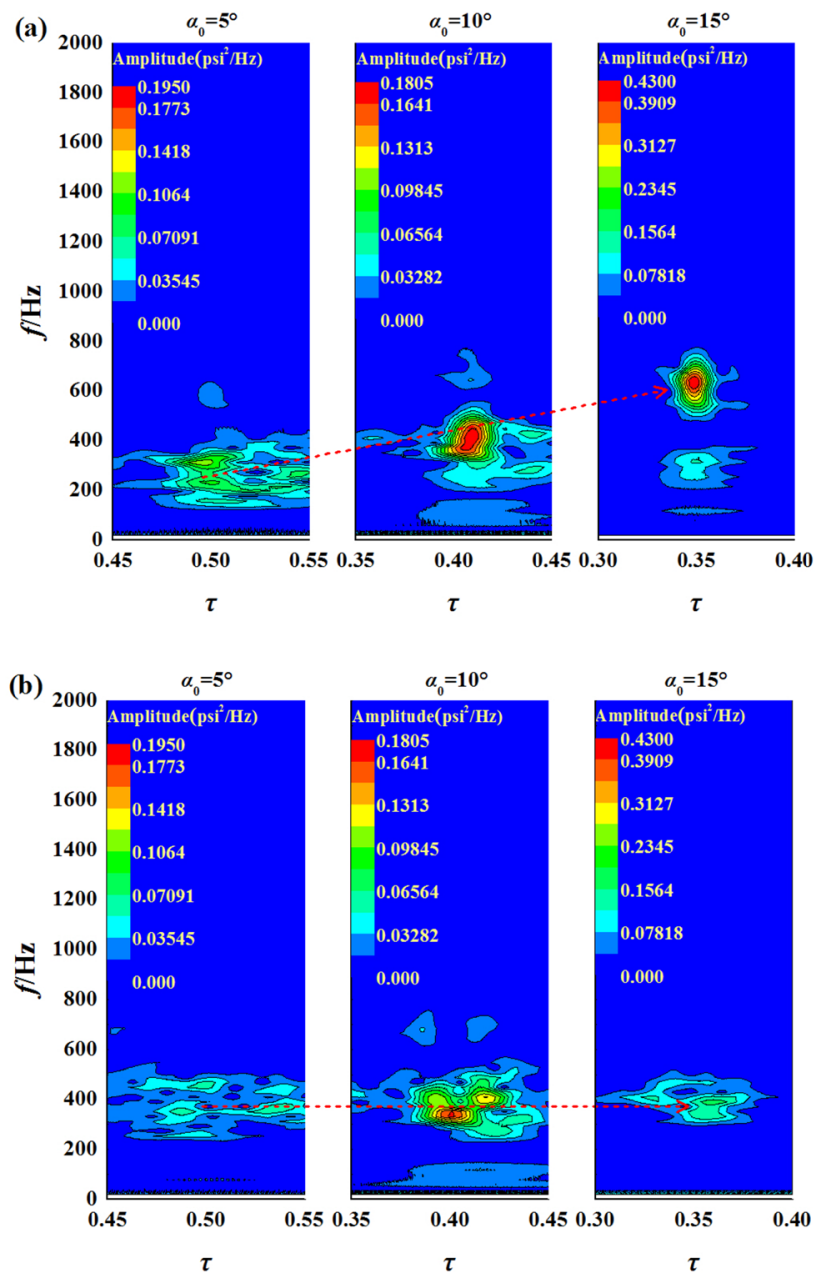

Fig. 23 Effects of mean AoA on DSV characteristic frequency. a $x / c=0.10 . \mathbf{b} x / c=0.18$

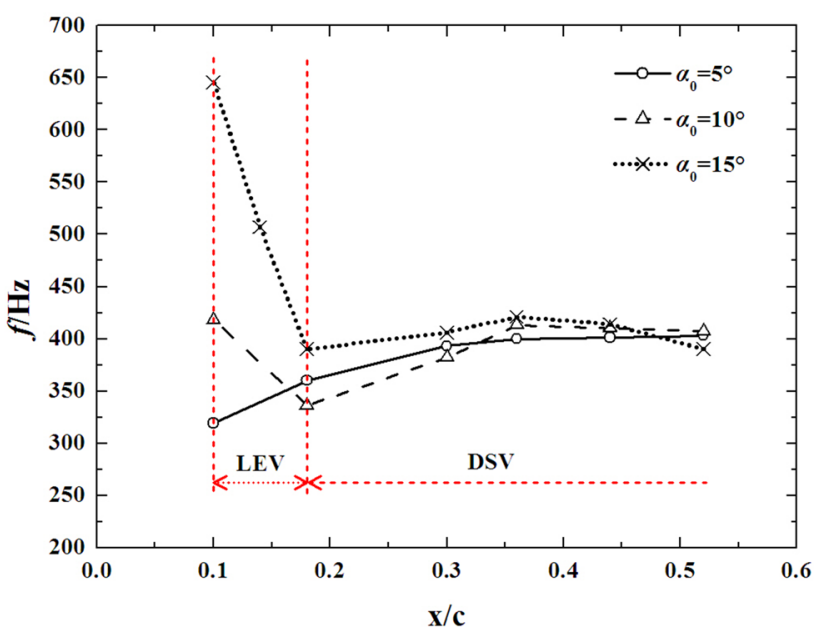

Fig. 24 Effects of mean AoA on DSV characteristic frequency 

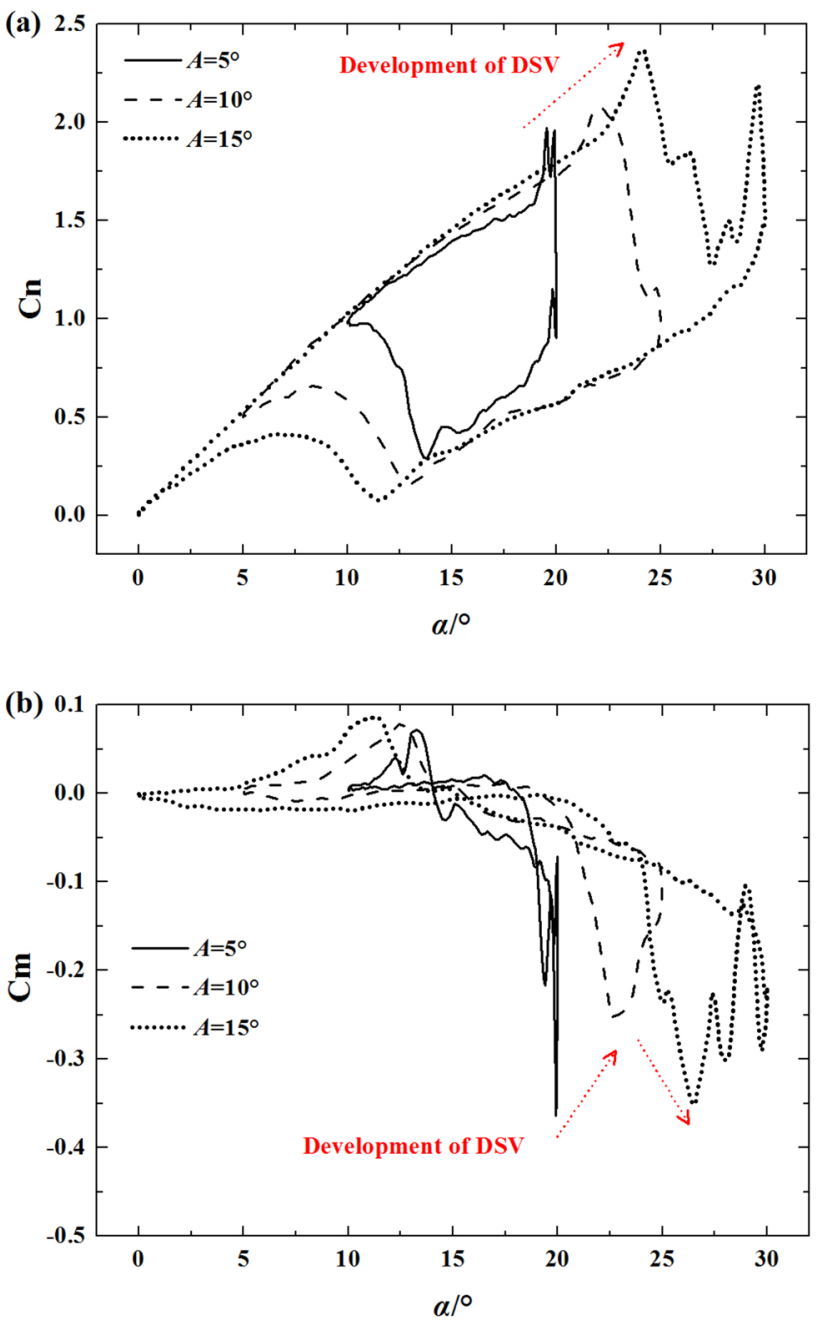

Fig. 25 Effects of amplitude on aerodynamic force and moment coefficients. a $\mathrm{Cn}$. b $\mathrm{Cm}$

The effects of the amplitude of the AoA on the DSV strength are shown in Fig. 26. With the increase in the amplitude, the strength of the DSV near the wall first decreased and then increased, and the effect of the amplitude on the strength of the DSV caused the moment coefficient to first increase and then decrease, as shown in Fig. 25. The strength of the DSV directly affected the variation of the minimum moment coefficient.

The effects of the amplitude of the AoA on the near-wall velocity of the DSV are shown in Fig. 27. In contrast to the effects of the mean AoA, the propagation velocity of the DSV along the near-wall surface first decreased and then increased with the increase in the amplitude of the AoA, showing significant nonlinearity. When the amplitude of the AoA was small $\left(A=5^{\circ}\right)$, the propagation velocity of the DSV along the suction surface of the airfoil was $V=12.59 \mathrm{~m} / \mathrm{s}$. As the amplitude increased to $A=10^{\circ}$, the propagation velocity gradually decreased to $V=9.03 \mathrm{~m} / \mathrm{s}$. When the amplitude

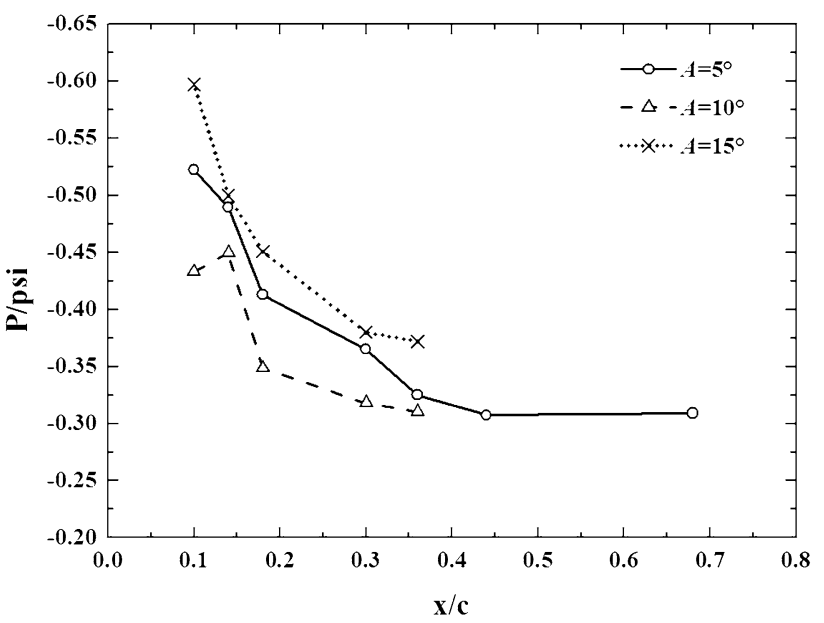

Fig. 26 Effects of amplitude on DSV strength

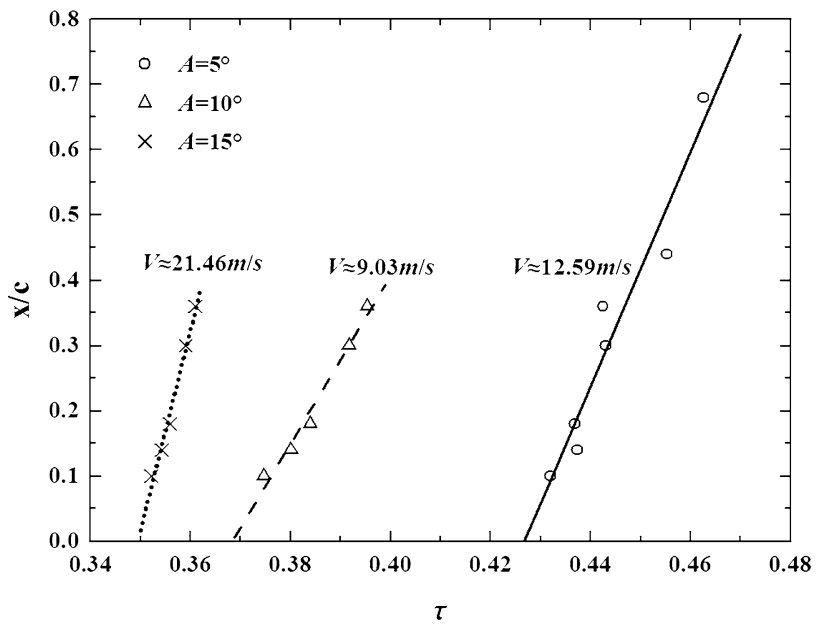

Fig. 27 Effects of amplitude on DSV evolution velocity

further increased to $A=15^{\circ}$, the propagation velocity of the DSV along the airfoil wall increased to $V=21.46 \mathrm{~m} / \mathrm{s}$.

Considering the data in Sect. 3.4, the DSV propagation speeds were $V=9.03-21.46 \mathrm{~m} / \mathrm{s}$ in this study. The free flow with a speed of $U=31.7 \mathrm{~m} / \mathrm{s}$ was used to perform the normalization, and the normalized speeds were $0.28 U-0.68 U$. McCroskey et al. [14] studied the DSV of the NACA0012 airfoil using the surface pressure, hot wires, and pitot tubes, and found that the propagation speed was about $0.35 U-0.4 U$. Lorber and Carta [15] also obtained the DSV propagation speed of SSC-A09 airfoil using the surface pressure, which was $0.16 U$. Chandrasekhara and Carr [16] and Zakaria et al. [17] used flow visualization technology to obtain the DSV propagation velocity of the NACA0012 airfoil, which was $0.3 U$. Wang and Zhao [18] studied the DSV on the OA209 and SC1095 airfoils and found that the DSV propagation speed was $0.4 U$, which had little relation to the airfoil shape. They also found that the 


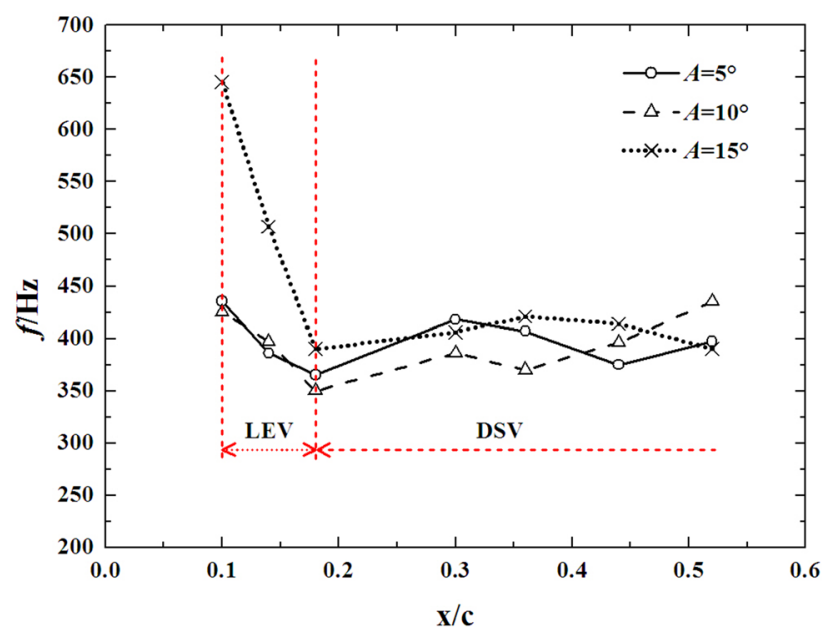

Fig. 28 Effects of amplitude of AoA on DSV characteristic frequency

reduced frequency affected the propagation speed. The results of this article were consistent with these previous results. In addition, we found that both the average AoA and amplitude of the AoA could affect the propagation speed of the DSV.

The effects of the amplitude of the AoA on the characteristic frequency of the near-wall DSV is shown in Fig. 28. At the initial stage of DSV development $(x / c=0.10-0.20)$, the characteristic frequency of DSV under the three amplitudes all decreased. The characteristic frequencies for $A=5^{\circ}$ and $A=10^{\circ}$ were very close, and they were significantly smaller than that for $A=15^{\circ}$. At the later stage of DSV development $(x / c>0.20)$, the amplitude had little effect on the characteristic frequency of the DSV, and the characteristic frequency remained at $f \approx 400 \mathrm{~Hz}$, which was consistent with the effect of the average AoA.

\section{Conclusion}

The physical characteristics of the DSV on the NACA0012 airfoil during its pitching oscillation were experimentally studied using surface pressure measurements with high time accuracy. The experimental Reynolds number was $\operatorname{Re}=1.5 \times 10^{6}$, and the reduced frequency was $k=0.069$. The propagation of the unsteady pressure field during the dynamic stall process was described in detail. This study focused on the DSV's motion characteristics, including its near-wall development characteristics and near-wall evolution velocity. Moreover, the frequency characteristics of the near-wall DSV were studied using wavelet analysis combined with POD technology. In addition, the effects of the mean AoA and amplitude on the DSV motion and frequency characteristics were discussed in detail.

In a typical dynamic stall process $\left(\alpha_{0}=15^{\circ}, A=15^{\circ}\right)$, a first LEV, called LEV1, was formed in the front part of the airfoil $(x / c=0.20-0.30)$, and then a second LEV, called LEV2, was rapidly formed at the leading edge of the airfoil $(x / c=0.10)$. As the AoA increased, the LEV gradually developed to the trailing edge, and formed the DSV. During the development of the DSV, its near-wall strength gradually decayed, indicating that the DSV gradually detached from the airfoil surface. At the early stage of the DSV development, the characteristic frequency was attenuated from $f \approx 600 \mathrm{~Hz}$ to $f \approx 400 \mathrm{~Hz}$. When the DSV developed to a certain position, the characteristic frequency of the DSV remained almost unchanged, which was $f \approx 400 \mathrm{~Hz}$.

The mean AoA had a significant effect on the physical properties of the DSV. Its influence on the DSV strength and propagation velocity near the wall was almost linear. With the increase in the average AoA, the strength of the near-wall DSV gradually increased, and its propagation velocity along the wall gradually increased from $0.36 U$ to $0.68 U$. The average AoA had a significant effect on the characteristic frequency of the LEV at the initial stage of DSV development $(x / c=0.10-0.20)$. With the increase in the AoA, the characteristic frequency of the LEV increased gradually. In the later stage of DSV development $(x / c>0.20)$, the average AoA had little effect on the characteristic frequency of the DSV, which remained at $f \approx 400 \mathrm{~Hz}$.

The amplitude of the AoA also had a significant effect on the physical properties of the DSV. In contrast to the effect of the mean AoA, the effects of the amplitude on the DSV strength and propagation velocity near the wall were nonlinear. With the increase in the amplitude, the strength and the propagation velocity of the DSV near the wall first decreased and then increased. At the initial stage of DSV development $(x / c=0.10-0.20)$, the DSV characteristic frequencies of all three amplitudes $\left(A=5^{\circ}, A=10^{\circ}\right.$, and $A=15^{\circ}$ ) gradually decreased. The characteristic frequencies at $A=5^{\circ}$ and $A=10^{\circ}$ were very close, and both were significantly smaller than that at $A=15^{\circ}$. In the later stage of DSV development $(x / c>0.20)$, the amplitude had little effect on the characteristic frequency of the DSV, and the characteristic frequency remained at $f \approx 400 \mathrm{~Hz}$, which was consistent with the effect of the mean AoA.

This study focused on the DSV properties under high Reynolds numbers, including the motion characteristics and the time-frequency characteristics. The effects of the mean AoA and the amplitude on the DSV properties were emphasized, allowing the dynamic stall phenomenon to be understood more deeply. This study has a certain guiding significance for flow control methods in different cases.

Acknowledgements We would like to thank to all the experimental staff of the NF-3 Wind Tunnel for their hard work. 
Funding This research did not receive any specific grant from funding agencies in the public, commercial, or not-for-profit sectors.

\section{Declarations}

Conflict of interest The authors declare that they have no conflict of interest. No conflict of interest exits in the submission of this manuscript, and manuscript is approved by all authors for publication. I would like to declare on behalf of my co-authors that the work described was original research that has not been published previously, and not under consideration for publication elsewhere, in whole or in part. All the authors listed have approved the manuscript that is enclosed.

Open Access This article is licensed under a Creative Commons Attribution 4.0 International License, which permits use, sharing, adaptation, distribution and reproduction in any medium or format, as long as you give appropriate credit to the original author(s) and the source, provide a link to the Creative Commons licence, and indicate if changes were made. The images or other third party material in this article are included in the article's Creative Commons licence, unless indicated otherwise in a credit line to the material. If material is not included in the article's Creative Commons licence and your intended use is not permitted by statutory regulation or exceeds the permitted use, you will need to obtain permission directly from the copyright holder. To view a copy of this licence, visit http://creativecommons.org/licenses/by/4.0/.

\section{References}

1. Carr LW (1988) Progress in analysis and prediction of dynamic stall. J Aircr 25(1):6-17. https://doi.org/10.2514/3.45534

2. Carr LW, McAlister KW (1983) The effect of a leading-edge slat on the dynamic stall of an oscillating airfoil. Aircraft Design Technol Meet. https://doi.org/10.2514/6.1983-2533

3. Carr LW, McAlister KW, McCroskey WJ (1977) Analysis of the development of dynamic stall based on oscillating airfoil experiments. IN: NASA TN-D-8382

4. McAlister KW, Carr LW, McCroskey WJ (1978) Dynamic stall experiments on the NACA0012 airfoil. In: NASA TP-1100

5. Chandrasekhara MS, Carr LW, Wilder MC (1994) Interferometric investigations of compressible dynamic stall over a transiently pitching airfoil. AIAA J 32(3):586-593. https://doi.org/10.2514/3. 12025

6. Chandrasekhara MS, Ahmed S, Carr LW (1993) Schlieren studies of compressibility effects on dynamic stall of transiently pitching airfoils. J Aircr 30(2):213-220. https://doi.org/10.2514/3.48268

7. Mulleners K, Henning A, Mai $\mathrm{H}$ et al (2009) Investigation of unsteady flow development over a pitching airfoil by means of TR-PIV. In: 27th AIAA Applied Aerodynamics. https://doi.org/ 10.2514/6.2009-3504

8. Wei BB, Gao YW, Wng L et al (2019) Analysis of flow transition and separation on oscillating airfoil by pressure signature. J Mech Sci Technol 33(1):279-288. https://doi.org/10.1007/ s12206-018-1227-0

9. Gupta R, Ansell PJ (2019) Unsteady flow physics of airfoil dynamic stall. AIAA J 57(1):165-175. https://doi.org/10.2514/1. J057257

10. Zou T, Lee C (2019) Rotor boundary layer development in a twostage compressor. Phys Fluids 31:123606. https://doi.org/10. $1063 / 1.5131805$

11. Jia L, Zou T, Zhu Y et al (2018) Rotor boundary layer development with inlet guide vane (IGV) wake impingement. Phys Fluids 30(4):040911. https://doi.org/10.1063/1.5013303
12. Pruski BJ, Bowersox RDW (2013) Leading-edge flow structure of a dynamically pitching NACA 0012 airfoil. AIAA J 51(5):1042_1053. https://doi.org/10.2514/1.J051673

13. Acharya M, Metwally M (1992) Unsteady pressure field and vorticity production over a pitching airfoil. AIAA J 30(2):403-411. https://doi.org/10.2514/3.10931

14. McCroskey WJ, Carr LW, McAlister KW (1976) Dynamic stall experiments on oscillating airfoils. AIAA J 14(1):57-63. https:// doi.org/10.2514/3.61332

15. Lorber PF, Carta FO (1988) Airfoil dynamic stall at constant pitch rate and high Reynolds number. J Aircr 25(6):548-556. https:// doi.org/10.2514/3.45621

16. Chandrasekhara MS, Carr LW (1990) Flow visualization studies of the Mach number effects on dynamic stall of an oscillating airfoil. J Aircr 27(6):516-522. https://doi.org/10.2514/3.25313

17. Zakaria MY, Taha HE, Hajj MR (2017) Measurement and modeling of lift enhancement on plunging airfoils: a frequency response approach. J Fluids Struct 69:187-208. https://doi.org/10.1016/j. jfluidstructs.2016.12.004

18. Wang Q, Zhao Q (2016) Experiments on unsteady vortex flowfield of typical rotor airfoils under dynamic stall conditions. Chin J Aeronaut 29(2):358-374. https://doi.org/10.1016/j.cja.2016.02.013

19. Karim M, Acharya M (1993) Control of the dynamic-stall vortex over a pitching airfoil by leading-edge suction. In: 3rd Shear Flow conference. https://doi.org/10.2514/6.1993-3267

20. Geissler W, Dietz G, Mai H et al (2005) Dynamic stall and its passive control investigations on the OA209 airfoil section. In: 31st European Rotorcraft Forum, Florence, Italy

21. Ajalli F, Mani M (2014) Effects of adding strip flap on a plunging airfoil. Aircr Eng Aerosp Technol 86(1):6-18. https://doi.org/10. 1108/AEAT-08-2012-0129

22. Geissler W, Dietz G, Mai H (2005) Dynamic stall on a supercritical airfoil. Aerosp Sci Technol 9(5):390-399. https://doi.org/10. 1016/j.ast.2005.01.012

23. Geissler W, Haselmeyer H (2006) Investigation of dynamic stall onset. Aerosp Sci Technol 10(7):590-600. https://doi.org/10. 1016/j.ast.2006.05.001

24. Worasinchai S, Ingram G, Dominy R (2013) Unsteady surface pressures and airload of a pitching airfoil. Energy Procedia 34:407-416. https://doi.org/10.1016/j.egypro.2013.06.769

25. Bourdet S, Braza M, Hoarau Y et al (2012) Prediction and physical analysis of unsteady flows around a pitching airfoil with the dynamic mesh approah. Eur J Comput Mech 16(3-4):451-476. https://doi.org/10.3166/REMN.16.451-476

26. Choudhuri PG, Knight DD (1996) Effects of compressibility, pitch rate and Reynolds number on unsteady incipient boundary layer separation over a pitching airfoil. J Fluid Mech 308(1):195-217. https://doi.org/10.1017/S0022112096001450

27. Sharma DM, Poddar K (2010) Investigations on quasi-steady characteristics for an airfoil oscillating at low reduced frequencies. Int J Aerosp Eng 2010:61-611. https://doi.org/10.1155/2010/940528

28. Li Z, F L et al (2019) Experimental and numerical investigation of three-dimensional vortex structures of a pitching airfoil at a transitional Reynolds number. Chin J Aeronaut 10:2254-2266

29. Patte-rouland B, Lalizei G, Moreau J et al (2001) Flow analysis of an annular jet by particle image velocimetry and proper orthogonal decomposition. Meas Sci Technol 12(9):1404-1412

30. Pedersen JM, Meyer KE (2002) POD analysis of flow structures in a scale model of a ventilated room. Exp Fluids 33:940-949. https://doi.org/10.1007/s00348-002-0514-8

31. Sirovich L (1987) Turbulence and the dynamics of coherent structures part I: coherent structures. Q Appl Math 45(3):561-571

Publisher's Note Springer Nature remains neutral with regard to jurisdictional claims in published maps and institutional affiliations. 